1990-06-01

\title{
Eigenvalue problems of Ginzburg-Landau operator in bounded domains
}

Kening Lu

Xing-Bin Pan

Follow this and additional works at: https://scholarsarchive.byu.edu/facpub

Part of the Mathematics Commons

\section{Original Publication Citation}

Lu, Kening and Xing B. Pan."Eigenvalue problems of Ginzburg-Landau operator in bounded domains." Journal of Mathematical Physics 4 (1999): 2647-267.

\section{BYU ScholarsArchive Citation}

Lu, Kening and Pan, Xing-Bin, "Eigenvalue problems of Ginzburg-Landau operator in bounded domains" (1990). Faculty Publications. 730.

https://scholarsarchive.byu.edu/facpub/730

This Peer-Reviewed Article is brought to you for free and open access by BYU ScholarsArchive. It has been accepted for inclusion in Faculty Publications by an authorized administrator of BYU ScholarsArchive. For more information, please contact ellen_amatangelo@byu.edu. 


\section{Eigenvalue problems of Ginzburg-Landau operator in bounded domains}

Kening Lu

Department of Mathematics, Brigham Young University, Provo, Utah 84602

Xing-Bin Pan

Center for Mathematical Sciences, Zhejiang University,

Hangzhou 310027, People's Republic of China

(Received 21 September 1998; accepted for publication 5 March 1999)

In this paper we study the eigenvalue problems for the Ginzburg-Landau operator with a large parameter in bounded domains in $\mathbb{R}^{2}$ under gauge invariant boundary conditions. The estimates for the eigenvalues are obtained and the asymptotic behavior of the associated eigenfunctions is discussed. These results play a key role in estimating the critical magnetic field in the mathematical theory of superconductivity. (C) 1999 American Institute of Physics. [S0022-2488(99)02806-6]

\section{INTRODUCTION AND STATEMENT OF MAIN RESULTS}

This paper is devoted to the asymptotic estimates, for large parameter $\sigma$, of the first eigenvalue $\mu(\sigma A)$ and the associated eigenfunctions of the Ginzburg-Landau operator $-\nabla_{\sigma A}^{2}$ in a smooth bounded domain $\Omega$ in $\mathbb{R}^{2}$. Given a real vector field $A=\left(A^{1}, A^{2}\right)$, the Ginzburg-Landau operator $-\nabla_{A}^{2}$ associated with $A$ is defined by

$$
-\nabla_{A}^{2} \psi=-\nabla_{A} \cdot\left(\nabla_{A} \psi\right)=-\nabla \psi+i[2 A \cdot \nabla \psi+\psi \operatorname{div} A]+|A|^{2} \psi
$$

where $i=\sqrt{-1}$. We denote $\nabla_{A} \psi=\nabla \psi-i \psi A, \operatorname{curl} A=\partial_{1} A^{2}-\partial_{2} A^{1}$, and $\operatorname{curl}^{2} A=\left(\partial_{2}(\operatorname{curl} A)\right.$, $\left.-\partial_{1}(\operatorname{curl} A)\right)$, here $\partial_{j}=\partial / \partial x_{j}$.

Let $\mu=\mu(A)$ be the first eigenvalue of the following problem:

$$
\begin{gathered}
-\nabla_{A}^{2} \psi=\mu \psi \quad \text { in } \Omega, \\
\left(\nabla_{A} \psi\right) \cdot \nu+\gamma \psi=0 \quad \text { on } \partial \Omega,
\end{gathered}
$$

where $\psi$ is a complex-valued function, $\nu$ is the unit outer normal to $\partial \Omega$, and $\gamma \geqslant 0$ is a given constant. Then,

$$
\mu(A)=\inf _{\psi \in W^{1,2}(\Omega)} \frac{\int_{\Omega}\left|\nabla_{A} \psi\right|^{2} d x+\gamma \int_{\partial \Omega}|\psi|^{2} d s}{\int_{\Omega}|\psi|^{2} d x} .
$$

It is well-known that the Ginzburg-Landau operator has the gange invariance property

$$
\nabla_{A+\nabla_{\chi}}\left(e^{i \chi} \psi\right)=e^{i \chi} \nabla_{A} \psi, \quad \nabla_{A+\nabla_{\chi}}^{2}\left(e^{i \chi} \psi\right)=e^{i \chi} \nabla_{A}^{2} \psi
$$

for every real smooth function $\chi$. The equation and the boundary condition in (1.1) as well as the functional in (1.2) are invariant under the gauge transformation $A \rightarrow A+\nabla_{\chi}, \psi \rightarrow e^{i \chi} \psi$. Therefore, $\mu\left(A+\nabla_{\chi}\right)=\mu(A)$. By a gauge transformation if necessary, we may assume

$$
\operatorname{div} A=0 \quad \text { in } \Omega, \quad A \cdot \nu=0 \quad \text { on } \partial \Omega .
$$

Our main result is the following 
Theorem 1: There exists a universal constant $\beta_{0}, 0<\beta_{0}<1$, such that for all $A \in C^{2}(\bar{\Omega})$

$$
\lim _{\sigma \rightarrow \infty} \frac{\mu(\sigma A)}{|\sigma|}=\underset{x \in \Omega}{\min }\left\{\min |\operatorname{curl} A(x)|, \beta_{0} \min _{x \in \partial \Omega}|\operatorname{curl} A(x)|\right\} .
$$

Remark 1.1: As a consequence of Theorem 1 we see that, if $\operatorname{curl} A(x) \equiv H$, a nonzero constant, then

$$
\lim _{\sigma \rightarrow \infty} \frac{\mu(\sigma A)}{|\sigma|}=\beta_{0}|H|
$$

The universal constant $\beta_{0}$ stated in Theorem 1 is the number $\beta(1)$ given in Lemma 2.2. We have an estimate for $\beta_{0}$ :

$$
0.5<\beta_{0}<0.76
$$

see Ref. 1. It has been expected that $\beta_{0} \simeq 0.59$, see for instance Refs. 2 and 3. If $\operatorname{curl} A$ vanishes at some points, the estimates can be greatly improved, see Sec. VI. It is interesting to see that the distribution of minimum points of $|\operatorname{curl} A|$ determines the magnitude of $\mu(\sigma A)$ and the location of the concentration points of the eigenfunctions for large $\sigma$. This is partially due to the gauge invariance of the Ginzburg-Landau operator and due to the invariance of $\operatorname{curl} A$ under the gauge transformations.

To prove Theorem 1 we shall establish two estimates for $\mu(\sigma A)$, the upper bound estimate (given in Sec. VI) and the lower bound estimate (given in Sec. VII). The gauge invariance of the Ginzburg-Landau operator, the local decomposition formula of vector fields obtained in Sec. III, and the results obtained in Ref. 4 concerning the eigenvalue problems of Ginzburg-Landau operator in the entire plane and on the half plane will play essential roles to obtain these estimates. To derive the lower bound estimate we also need to show the local convergence, as $\sigma \rightarrow \infty$, of the rescaled eigenfunctions (after a series of gauge transformations). Since the eigenfunctions may concentrate either in the interior of $\Omega$ or at the boundary, both interior and boundary a priori estimates established in Secs. IV and V are needed to obtain the local convergence. We mention that most of the estimates given in this paper are gauge invariant. As a by-product, the asymptotic behavior of the eigenfunctions as $\sigma$ goes to $\infty$ will also be obtained.

The technical difficulty in our problem comes from the boundary effects, which is our main concern in this paper. One may see in Sec. VI that when the eigenfunctions concentrate in the interior of $\Omega$, the limiting equation obtained after rescaling is an eigenvalue problem in the entire plane $R^{2}$, see (2.3). All the eigenvalues of (2.3) have been obtained in Ref. 4. However, when the concentration happens at the boundary, very technical analyses are required to get the boundary estimates and to prove the local convergence of the rescaled eigenfunctions near the boundary. In this case, the limiting equation is an eigenvalue problem in the half plane $\partial \mathbb{R}_{+}^{2}$, see (2.5). The first eigenvalue $\beta(h)$ of (2.5) was obtained in Ref. 4 after lengthy analyses, which is the difficult part of Ref. 4. Comparing Lemma 2.1 with Lemma 2.2 in Sec. II, one may see the significant difference between the problems in the domain without or with boundary.

The motivation to study such type eigenvalue problems is to estimate the value of the upper critical magnetic field at which superconductivity can nucleate.

In the mathematical theory of superconductivity, the following Ginzburg-Landau equation for $(\psi, A)$ was proposed as a macroscopic model (see Ref. 5)

$$
\begin{gathered}
-\nabla_{\kappa A}^{2} \psi=\kappa^{2}\left(1-|\psi|^{2}\right) \psi, \\
\operatorname{curl}^{2} A=-\frac{i}{2 \kappa}(\bar{\psi} \nabla \psi-\psi \nabla \bar{\psi})-|\psi|^{2} A+\operatorname{curl} H \quad \text { in } \Omega .
\end{gathered}
$$


Here $\Omega$ is the region occupied by the superconducting specimen, $\psi$ is a complex-valued function called order parameter, $A$ is a real vector field called magnetic potential, $H$ is the applied magnetic field, $\kappa$ is the so-called Ginzburg-Landau parameter. The natural boundary conditions for a superconductor-other material junction are (see Ref. 6)

$$
\left(\nabla_{\kappa A} \psi\right) \cdot \nu+\gamma \psi=0, \quad(\operatorname{curl} A-H) \times \nu=0 \quad \text { on } \partial \Omega,
$$

where $\nu$ is the unit out-normal vector at the boundary of $\Omega$ and $\gamma$ is a positive constant.

It is well-known that a superconductor placed in an applied magnetic field may change its phase when the field varies. Consider a spatially homogeneous field. If the field is sufficiently strong, it penetrates through the entire sample and the superconductor is in a normal state. As the field is gradually reduced to a certain value $H_{C_{3}}$ called the upper critical field, the nucleation of superconductivity at surface occurs. If the field is further reduced to another value $H_{C_{2}}$, the nucleation in the interior occurs. It is important in both theory and applications to estimate the values of the critical fields, especially for type 2 superconductors with large value of $\kappa$.

The physicists Saint-James and De Gennes were the first to study the surface nucleation phenomenon for semi-infinite superconductor occupying the half space (see Ref. 2). The most amazing result they obtained was the relation $H_{C_{3}} / H_{C_{2}}=1 / 0.59$. The argument for this relation was nontrivial, even though they studied only the superconductor which occupies the half space and is subjected to a spatially homogeneous applied magnetic field.

We have been interested in estimating the value of the upper critical field for superconducting specimen occupying an arbitrary bounded smooth domain. In Ref. 1, to get such estimate, we considered the applied field having the form $H=\sigma H_{0}$ and estimated the maximal value of $\sigma$, say $\sigma^{*}$, so that under the applied field $\sigma^{*} H_{0}$ the nucleation of superconductivity occurs. Choosing a vector field $F$ so that $\operatorname{curl} F=H_{0}$, we found that when $\kappa$ is large, the value of $\sigma^{*}$ is close to the number $\sigma_{*}$ for which $\mu(\sigma * \kappa F)=\kappa^{2}$. This led us to study the asymptotic estimates of $\mu(\sigma F)$ for large value of $\sigma$. In Ref. 1, by using the results in this paper, we obtained the asymptotic estimate for $H_{C_{3}}$ for large $\kappa$ and the location of nucleation of superconductivity.

There have been many recent works on the mathematical theory of superconductivity, see Refs. 3, 7-19, and the references therein. The works ${ }^{3}$ by Chapman, ${ }^{7}$ by Bauman, Phillips, and Tang, and by Bernoff and Sternberg ${ }^{10}$ are closely related to our present paper, while Refs. 7 and 10 were found after this work had been completed. In Ref. 3, Chapman studied the half-plane problem on $\mathrm{H}_{\mathrm{C}_{3}}$ by using formal mathematical analysis. In Ref. 7, Bauman, Phillips, and Tang rigorously estimated $H_{C_{3}}$ and found the location of nucleation for a sample occupying a cylinder with two-dimensional cross section consisting of a disk. The sample is adjacent to a vacuum and is subject to a homogeneous applied magnetic field pointing in the axial direction. From the bifurcation point of view, they studied small solutions bifurcating from the eigenfunctions. In Ref. 10, Bernoff and Sternberg considered a sample occupying an infinite cylinder with twodimensional cross section consisting of an arbitrary simply connected smooth bounded region in $\mathrm{R}^{2}$. The sample is adjacent to a vacuum and is subject to a homogeneous applied magnetic field pointing in the axial direction. They estimated $H_{C_{3}}$ and found the location of nucleation by using formal asymptotic expansions. In this paper we study eigenvalue problems in bounded smooth domains with nonhomogeneous applied magnetic fields under the boundary conditions for a superconductor-other material junction. The result obtained in this paper was used in Ref. 1 to obtain rigorously estimates for $H_{C_{3}}$ and locations of nucleation for a cylindrical sample which is placed in an applied magnetic field being parallel to the lateral surface but not necessarily spatially homogeneous and is adjacent to other material.

\section{PRELIMINARIES}

In this section we give some basic lemmas which will be used later to establish our main result. Throughout this paper, we let 


$$
\omega(x)=\frac{1}{2}\left(-x_{2}, x_{1}\right)
$$

Note that $\operatorname{curl} \omega=1$ and $\operatorname{div} \omega=0$. Denote, for a nonzero real number $h$,

$$
\alpha(h)=\inf _{\psi \in \mathcal{W}\left(\mathbb{R}^{2}\right)} \frac{\int_{\mathbb{R}^{2}}\left|\nabla_{h \omega} \psi\right|^{2} d x}{\int_{\mathbb{R}^{2}}|\psi|^{2} d x},
$$

where $\mathcal{W}\left(\mathbb{R}^{2}\right)=W_{\text {loc }}^{1,2}\left(\mathbb{R}^{2}\right) \cap L^{2}\left(\mathbb{R}^{2}\right)$. Clearly, the minimizers are the $L^{2}$ eigenfunctions of the following problem associated with $\alpha=\alpha(h)$ :

$$
-\nabla_{h \omega}^{2} \psi=\alpha \psi \text { in } \mathbb{R}^{2}
$$

Let

$$
\beta(h)=\inf _{\psi \in \mathcal{W}\left(\mathbb{R}_{+}^{2}\right)} \frac{\int_{\mathbb{R}_{+}^{2}}\left|\nabla_{h \omega} \psi\right|^{2} d x}{\int_{\mathbb{R}_{+}^{2}}|\psi|^{2} d x},
$$

where $\mathcal{W}\left(\mathbb{R}_{+}^{2}\right)=W_{\text {loc }}^{1,2}\left(\mathbb{R}_{+}^{2}\right) \cap L^{2}\left(\mathbb{R}_{+}^{2}\right)$. The associated eigenvalue problem is

$$
\begin{gathered}
-\nabla_{h \omega}^{2} \psi=\beta \psi \quad \text { in } \mathbb{R}_{+}^{2}, \\
\left(\nabla_{h \omega} \psi\right) \cdot \nu=0 \quad \text { on } \partial \mathbb{R}_{+}^{2},
\end{gathered}
$$

where $\nu(x)=(0,-1)$ is the outer normal to $\mathrm{R}_{+}^{2}$.

Lemma 2.1: (Ref. 4). For every $h \neq 0, \alpha(h)=|h|$. The associated eigenfunctions are given by

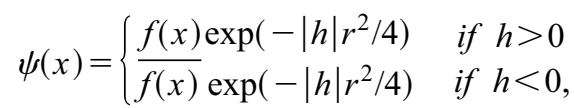

where $r=|x|, f(x)$ is any function analytic in $\mathbb{R}^{2}$ such that $f(x) \exp \left(-|h| r^{2} / 4\right) \in L^{2}\left(\mathbb{R}^{2}\right)$. For all $\alpha<\alpha(h)$, (2.3) has no nontrivial bounded solution.

Lemma 2.2: (Ref. 4). There exists a positive constant $\beta_{0}, 0<\beta_{0}<1-1 / \sqrt{2 e \pi}$, such that $\beta(h)=\beta_{0}|h|$. For all $h \neq 0, \beta(h)$ is not achieved in $\mathcal{W}\left(\mathbb{R}_{+}^{2}\right)$, i.e., there is no $L^{2}$ eigenfunction associated with $\beta(h)$. For all $\beta<\beta(h)$, (2.5) has no nontrivial bounded solution.

\section{LOCAL DECOMPOSITION OF VECTOR FIELDS}

In the proof of the convergence of the rescaled eigenfunctions in later sections, we use the gauge transformations frequently. Thus, we need to decompose a vector field into a gradient part and a curl part near a given point $P$. When $P$ is an interior point, this decomposition follows directly from the Taylor expansion (see Lemma 3.1). When $P$ is a boundary point, we need to decompose the vector field in new coordinates which straighten a portion of boundary (see Lemma 3.2).

Let $A(x)=\left(A^{1}(x), A^{2}(x)\right) \in C^{2}\left(\overline{B_{R}}\right)$ and denote

$$
a_{j}^{i}=\frac{\partial A^{i}}{\partial x_{j}}(0), \quad a_{j k}^{i}=\frac{\partial^{2} A^{i}}{\partial x_{j} \partial x_{k}}(0), \quad a^{1}=A^{1}(0), \quad a^{2}(0)=A^{2}(0) .
$$

Let $H(x)=\operatorname{curl} A(x)$. Then, $\operatorname{curl}^{2} A(x)=\left(\partial_{2} H,-\partial_{1} H\right)$.

Lemma 3.1: Let $A \in C^{2}\left(\overline{B_{R}}\right)$. Then,

$$
A(x)=A(0)+\nabla \xi(x)+\nabla \zeta(x)+\operatorname{curl} A(0) \omega(x)-\frac{1}{2}|x|^{2} \operatorname{curl}^{2} A(0)+D(x),
$$

where 


$$
\begin{gathered}
\xi(x)=\frac{1}{2}\left[a_{1}^{1} x_{1}^{2}+\left(a_{2}^{1}+a_{1}^{2}\right) x_{1} x_{2}+a_{2}^{2} x_{2}^{2}\right], \\
\zeta(x)=\frac{1}{6}\left[c_{1} x_{1}^{3}+3 c_{2} x_{1}^{2} x_{2}+3 c_{3} x_{1} x_{2}^{2}+c_{4} x_{2}^{3}\right]
\end{gathered}
$$

with

$$
c_{1}=a_{11}^{1}+\partial_{2} H(0), \quad c_{2}=a_{12}^{1}, \quad c_{3}=a_{12}^{2}, \quad c_{4}=a_{22}^{2}-\partial_{1} H(0),
$$

and $|D(x)|=o\left(|x|^{2}\right)$ as $x \rightarrow 0$. If $A \in C^{3}\left(\overline{B_{R}}\right)$, then $|D(x)| \leqslant C(R)|x|^{3}$ in $B_{R}$.

In the following we assume that $\Omega$ is a smooth (say, $C^{k}$ for some $k \geqslant 3$ ) bounded domain in $\mathrm{R}^{2}$ and $0 \in \partial \Omega$. Then, $\partial \Omega$ consists of a finite number of simple closed $C^{k}$ curves disintersecting with each other. Every component $\Gamma$ of $\partial \Omega$ can be represented as $z=z(s)$, where $s$ is the arclength of $\Gamma$, and $\tau(s)=\left(\tau_{1}, \tau_{2}\right)=z^{\prime}(s)$ is the unit tangent vector. Let $\nu(s)=\left(\nu_{1}, \nu_{2}\right)$ be the unit outer normal. We choose the positive direction of $\Gamma$ in such a way that the orientation of $(\nu, \tau)$ is coincident with the orientation of the $x_{1} x_{2}$ coordinates. Then, $\tau_{1}=-\nu_{2}, \tau_{2}=\nu_{1}$. From the Frenet formula we have

$$
\tau^{\prime}=-\kappa_{r} \nu, \quad \tau^{\prime \prime}=-\kappa_{r}^{\prime} \nu-\kappa_{r}^{2} \tau, \quad \nu^{\prime}=\kappa_{r} \tau, \quad \nu^{\prime \prime}=\kappa_{r}^{\prime} \tau-\kappa_{r}^{2} \nu
$$

where $\kappa_{r}$ is the relative curvature of $\Gamma$ under the given orientation. Obviously, there exists a positive constant $\mu_{0}=\mu_{0}(\Omega)$ such that $\left|\kappa_{r}\right| \leqslant 1 / \mu_{0}$ on $\partial \Omega$.

Fix $0<\mu<\mu_{0}$. Denote by $d(x)=\operatorname{dist}(x, \partial \Omega)$ the distance function, and denote $\Omega(\mu)=\{x$ $\in \overline{\Omega:} d(x)<\mu\}$. Then, $d \in C^{k-1}\left(\overline{\Omega\left(\mu_{0}\right)}\right)$. For every $x \in \Omega\left(\mu_{0}\right)$ there exists a unique point $z$ $=z(x) \in \partial \Omega$ such that $x=z-d(x) \nu(z), \nabla d(x)=-\nu(z)$. The mapping

$$
x=\mathcal{F}(s, t)=z(s)-t \nu(s)
$$

determines a $C^{1}$ transformation of coordinates. Set

$$
g(s, t)=|\operatorname{det} D \mathcal{F}|=\left|\mathcal{F}_{s} \times \mathcal{F}_{t}\right|=1-t \kappa_{r}(s) .
$$

After rotating the coordinate system we may assume $\tau(0)=(1,0), \nu(0)=(0,-1)$. Denote $\mathbf{e}_{1}=\tau, \mathbf{e}_{2}=-\nu, y_{1}=s, y_{2}=t, y=\left(y_{1}, y_{2}\right) . y$ is the new coordinate straightening the boundary. Using (3.3) we get

$$
\begin{gathered}
\mathbf{e}_{1}(y)=\tau\left(y_{1}\right)=\left(\begin{array}{l}
1 \\
0
\end{array}\right)+\left(\begin{array}{c}
0 \\
\kappa_{r}(0) y_{1}
\end{array}\right)+\frac{1}{2} y_{1}^{2}\left(\begin{array}{c}
-\kappa_{r}^{2}(0) \\
\kappa_{r}^{\prime}(0)
\end{array}\right)+O\left(\left|y_{1}\right|^{3}\right), \\
\mathbf{e}_{2}(y)=-\nu\left(y_{1}\right)=\left(\begin{array}{l}
0 \\
1
\end{array}\right)-\left(\begin{array}{c}
\kappa_{r}(0) y_{1} \\
0
\end{array}\right)-\frac{1}{2} y_{1}^{2}\left(\begin{array}{c}
\boldsymbol{\kappa}_{r}^{\prime}(0) \\
\kappa_{r}^{2}(0)
\end{array}\right)+O\left(\left|y_{1}\right|^{3}\right), \\
\mathcal{F}(y)=y+\frac{\kappa_{r}(0)}{2}\left(\begin{array}{c}
-2 y_{1} y_{2} \\
y_{1}^{2}
\end{array}\right)+O\left(|y|^{3}\right) \quad \text { as } y \rightarrow 0, \\
g(y)=1-\kappa_{r}(0) y_{2}-\kappa_{r}^{\prime}(0) y_{1} y_{2}+O\left(|y|^{3}\right) .
\end{gathered}
$$

Denote the inverse map of $\mathcal{F}$ by $\mathcal{G}(x)$. At the point $x=\mathcal{F}(y)$ we have

$$
D \mathcal{G}(x)=\left(\begin{array}{ll}
\mathcal{G}_{1}^{1} & \mathcal{G}_{2}^{1} \\
\mathcal{G}_{1}^{2} & \mathcal{G}_{2}^{2}
\end{array}\right)=\frac{1}{1-y_{2} \kappa_{r}\left(y_{1}\right)}\left(\begin{array}{cc}
-\nu_{2} & \nu_{1} \\
-\left(1-y_{2} \kappa_{r}\left(y_{1}\right)\right) \tau_{2} & \left(1-y_{2} \kappa_{r}\left(y_{1}\right)\right) \tau_{1}
\end{array}\right) .
$$

For a given vector field $A(x)$ we define a new vector field a(y) associated with $A(x)$ by

$$
\mathbf{a}(y)=\mathbf{a}^{1}(y) \mathbf{e}_{1}+\mathbf{a}^{2}(y) \mathbf{e}_{2},
$$


where

$$
\mathbf{a}^{1}(y)=g(y) A(\mathcal{F}(y)) \cdot \mathbf{e}_{1}(y), \quad \mathbf{a}^{2}(y)=A(\mathcal{F}(y)) \cdot \mathbf{e}_{2}(y) .
$$

Then,

$$
\begin{aligned}
\mathbf{a}^{1}(y)= & a^{1}+\left[a_{1}^{1}+\kappa_{r}(0) a^{2}\right] y_{1}+\left[a_{2}^{1}-\kappa_{r}(0) a^{1}\right] y_{2}+\frac{1}{2}\left\{\left[a_{11}^{1}+\kappa_{r}(0) a_{2}^{1}+2 \kappa_{r}(0) a_{1}^{2}\right.\right. \\
& \left.-\kappa_{r}(0)^{2} a^{1}+\kappa_{r}^{\prime}(0) a^{2}\right] y_{1}^{2}+2\left[a_{12}^{1}-2 \kappa_{r}(0) a_{1}^{1}+\kappa_{r}(0) a_{2}^{2}-\kappa_{r}^{2}(0) a^{2}-\kappa_{r}^{\prime}(0) a^{1}\right] y_{1} y_{2} \\
& \left.+\left[a_{22}^{1}-2 \kappa_{r}(0) a_{2}^{1}\right] y_{2}^{2}\right\}+o\left(|y|^{2}\right), \\
\mathbf{a}^{2}(y)= & a^{2}+\left(a_{1}^{2}-\kappa_{r}(0) a^{1}\right) y_{1}+a_{2}^{2} y_{2}+\frac{1}{2}\left\{\left[a_{11}^{2}+\kappa_{r}(0) a_{2}^{2}-2 \kappa_{r}(0) a_{1}^{1}-\kappa_{r}^{2}(0) a^{2}-\kappa_{r}^{\prime}(0) a^{1}\right] y_{1}^{2}\right. \\
& \left.+2\left[a_{12}^{2}-\kappa_{r}(0) a_{1}^{2}-\kappa_{r}(0) a_{2}^{1}\right] y_{1} y_{2}+a_{22}^{2} y_{2}^{2}\right\}+o\left(|y|^{2}\right) .
\end{aligned}
$$

Summarizing the above discussion, we obtain

Lemma 3.2: Let $\Omega$ be a smooth domain in $R^{2}$ with $0 \in \partial \Omega$. Assume that $A$ $\in C^{2}\left(\overline{\Omega \cap \mathcal{F}\left(B_{R}\right)}\right)$. Then, in the new coordinates $y$ straightening the boundary, the vector field $\mathbf{a}(y)$ associated with $A(x)$ has the following decomposition for $y \in B_{R}$ :

$$
\begin{aligned}
\left(\mathbf{a}^{1}(y), \mathbf{a}^{2}(y)\right)= & A(0)+\nabla \tilde{\xi}(y)+\nabla \tilde{\zeta}(y)+\operatorname{curl} A(0) \omega(y) \\
& -\frac{|y|^{2}}{2}\left[\operatorname{curl}^{2} A(0)-\kappa_{r}(0) \operatorname{curl} A(0) \tau(0)\right]+\widetilde{D}(y),
\end{aligned}
$$

where

$$
\begin{gathered}
\tilde{\xi}(y)=\frac{1}{2}\left[\left(a_{1}^{1}+\kappa_{r}(0) a^{2}\right) y_{1}^{2}+\left(a_{2}^{1}+a_{1}^{2}-2 \kappa_{r}(0) a^{1}\right) y_{1} y_{2}+a_{2}^{2} y_{2}^{2}\right], \\
\tilde{\zeta}(y)=\frac{1}{6}\left[\widetilde{c}_{1} y_{1}^{3}+3 \widetilde{c}_{2} y_{1}^{2} y_{2}+3 \widetilde{c}_{3} y_{1} y_{2}^{2}+\widetilde{c}_{4} y_{2}^{3}\right]
\end{gathered}
$$

with

$$
\begin{gathered}
\tilde{c}_{1}=a_{11}^{1}-a_{22}^{1}+a_{12}^{2}+\kappa_{r}(0)\left(a_{1}^{2}+a_{2}^{1}\right)-\kappa_{r}^{2}(0) a^{1}+\kappa_{r}^{\prime}(0) a^{2}, \\
\tilde{c}_{2}=a_{12}^{1}+\kappa_{r}(0)\left(a_{2}^{2}-2 a_{1}^{1}\right)-\kappa_{r}^{2}(0) a^{2}-\kappa_{r}^{\prime}(0) a^{1}, \\
\tilde{c}_{3}=a_{12}^{2}-\kappa_{r}(0)\left(a_{1}^{2}+a_{2}^{1}\right), \\
\tilde{c}_{4}=a_{12}^{1}-a_{11}^{2}+a_{22}^{2} .
\end{gathered}
$$

$|\widetilde{D}(y)|=o\left(|y|^{2}\right)$ as $y \rightarrow 0$. If $A \in C^{3}\left(\overline{\Omega \cap \mathcal{F}\left(B_{R}\right)}\right)$, then $|\widetilde{D}(y)| \leqslant C(R)|y|^{3}$ in $B_{R}^{+}$.

Note that in (3.9) $\nabla \widetilde{\xi}(y)=\left(\partial_{y_{1}} \widetilde{\xi}, \partial_{y_{2}} \widetilde{\xi}\right)$. In the following we denote

$$
\nabla_{y} f=\left(\frac{\partial f}{\partial y_{1}}\right) \mathbf{e}_{1}+\left(\frac{\partial f}{\partial y_{2}}\right) f \mathbf{e}_{2}
$$

From (3.7) and (3.10), we can write (3.9) as follows:

$$
\begin{aligned}
\mathbf{a}(y)= & A(0)+\nabla_{y} \tilde{\xi}(y)+\nabla_{y} \widetilde{\zeta}(y)+\operatorname{curl} A(0) \widetilde{\omega}(y) \\
& -\frac{|y|^{2}}{2}\left[\operatorname{curl}^{2} A(0)-\kappa_{r}(0) \operatorname{curl} A(0) \tau(0)\right]+\widetilde{D}(y),
\end{aligned}
$$


where $\widetilde{\omega}(y)=-\left(y_{2} / 2\right) \mathbf{e}_{1}+\left(y_{1} / 2\right) \mathbf{e}_{2}$. The decomposition in the form of (3.11) is more closely related to the gauge invariance of the operators involving the vector a, and will be used often in later sections.

\section{INTERIOR ESTIMATES}

In this section we shall derive a priori interior estimates for the solutions $\psi$ of the equation

$$
\nabla_{A}^{2} \psi=g \quad \text { in } \Omega,
$$

where the vector field $A$ and the function $g$ are given. We shall establish the gauge invariant estimates which depend on $\operatorname{curl} A$ instead of $A$ itself.

Theorem 4.1: Assume that $\psi$ is a smooth solution of $E q$. (4.1) and $\operatorname{curl} A \in L^{2}(\Omega)$. Then, for any compact subset $K$ of $\Omega$, there exists a constant $C$ depending only on $\Omega$ and $K$ such that

$$
\begin{aligned}
\left\|\left|\nabla_{A} \psi\right|\right\|_{H^{1}(K)}^{2} \leqslant \sum_{j, k}\left\|\nabla_{A^{j}} \nabla_{A^{k}} \psi\right\|_{L^{2}(K)}^{2} \leqslant & 2\|\psi \operatorname{curl} A\|_{L^{2}(\Omega)}^{2}+6\|g\|_{L^{2}(\Omega)}^{2}+C\left[1+\|\operatorname{curl} A\|_{L^{2}(\Omega)}^{4}\right] \\
& \times\left[\left\|\nabla_{A} \psi\right\|_{L^{2}(\Omega)}^{2}+\|\psi\|_{L^{2}(\Omega)}^{2}\right]
\end{aligned}
$$

Before proving Theorem 4.1 we mention that $\left\|\nabla_{A} \psi\right\|_{L^{2}}$ can be controlled by $\|\psi\|_{L^{2}}$, as shown in the following

Lemma 4.2: Assume that $\psi \in W_{\mathrm{loc}}^{1,2}(\Omega)$ is a weak solution of Eq. (4.1) for $g \in L_{\mathrm{loc}}^{2}(\Omega)$. Then, for every $R>0$ such that $B_{2 R} \subset \Omega$ we have

$$
\int_{B_{R}}\left|\nabla_{A} \psi\right|^{2} d x \leqslant 2 \int_{B_{2 R}}|g \psi| d x+\frac{16}{R^{2}} \int_{R_{2 R}}|\psi|^{2} d x
$$

Proof: Let $\eta$ be a smooth cutoff function supported in $B_{2 R}$ such that $\eta=1$ on $B_{R}$ and $|\nabla \eta|$ $\leqslant 2 / R$. Multiplying Eq. (4.1) by $\eta^{2} \psi$ and integrating by parts we get the conclusion.

For convenience we denote $F_{j, k} \psi=\left(\nabla_{A^{j}} \nabla_{A^{k}}-\nabla_{A^{k}} \nabla_{A^{j}}\right) \psi, \nabla_{A^{j}} \psi=\left(\partial_{j}-i A^{j}\right) \psi$ and $(\phi, \psi)$ $=\int_{\Omega} \phi \bar{\psi} d x$.

Proposition 4:3: Let $A \in C^{1}(\bar{\Omega})$ and $\psi \in C^{2}(\bar{\Omega})$. Then,

$$
\sum_{j, k}\left\|\nabla_{A^{j}} \nabla_{A^{k}} \psi\right\|_{L^{2}(\Omega)}^{2}=\|\psi \operatorname{curl} A\|_{L^{2}(\Omega)}^{2}+\left\|\nabla_{A}^{2} \psi\right\|_{L^{2}(\Omega)}^{2}+2 \mathfrak{F}\left(\overline{\nabla_{A^{1}} \psi} \nabla_{A^{2}} \psi, \operatorname{curl} A\right)+I(\partial \Omega),
$$

where

$$
\begin{aligned}
I(\partial \Omega) & =\int_{\partial \Omega}\left\{\sum_{j, k}\left[\overline{\nabla_{A^{k}} \nabla_{A^{j}} \psi} \nabla_{A^{k}} \psi \nu_{j}\right]-\overline{\nabla_{A}^{2} \psi}\left(\nabla_{A} \psi\right) \cdot \nu\right\} d s \\
& =\int_{\partial \Omega}\left\{\frac{1}{2} \frac{\partial}{\partial \nu}\left|\nabla_{A} \psi\right|^{2}+(\operatorname{curl} A)\left[\mathfrak{F}(\bar{\psi} \nabla \psi)-|\psi|^{2} A\right] \cdot \tau-\mathfrak{R}\left(\nabla_{A} \psi \cdot \nu\right) \overline{\nabla_{A}^{2} \psi}\right\} d s .
\end{aligned}
$$

Here $\tau$ is the unit tangent vector to $\partial \Omega$ such that the orientation of $(\nu, \tau)$ is the same as the orientation of $x_{1} x_{2}$ coordinates.

Proof: Let $\psi_{j}=\nabla_{A^{j}} \psi$. Then,

$$
\begin{aligned}
\left(\nabla_{A^{j}} \nabla_{A^{k}} \psi, \nabla_{A^{j}} \nabla_{A^{k}} \psi\right)= & \left(\nabla_{A^{j}} \psi_{k}, F_{j, k} \psi\right)-\left(\psi_{k}, F_{j, k} \psi_{j}\right)+\left(\nabla_{A^{k}} \psi_{k}, \nabla_{A^{j}} \psi_{j}\right) \\
& +\int_{\partial \Omega} \overline{\nabla_{A^{k}} \psi_{j}} \psi_{k} \nu_{j} d s-\int_{\partial \Omega} \overline{\nabla_{A^{j}} \psi_{j}} \psi_{k} \nu_{k} d s
\end{aligned}
$$


Taking summation over $1 \leqslant j, k \leqslant 2$ we obtain

$$
\begin{aligned}
& \sum_{j, k}\left\|\nabla_{A^{j}} \nabla_{A^{k}} \psi\right\|_{L^{2}(\Omega)}^{2}=\sum_{j, k}\left[\left(\nabla_{A^{j}} \psi_{k}, F_{j, k} \psi\right)-\left(\psi_{k}, F_{j, k} \psi_{j}\right)\right]+\left\|\nabla_{A}^{2} \psi\right\|_{L^{2}(\Omega)}^{2} \\
& +\int_{\partial \Omega}\left\{\sum_{j, k}\left[\overline{\nabla_{A} k \psi_{j}} \psi_{k} \nu_{j}\right]-\overline{\nabla_{A}^{2} \psi}\left(\nabla_{A} \psi\right) \cdot \nu\right\} d s \\
& =i\left(\bar{\psi}\left[\nabla_{A^{1}} \psi_{2}-\nabla_{A^{2}} \psi_{1}\right]+\psi_{1} \overline{\psi_{2}}-\psi_{2} \overline{\psi_{1}}, \operatorname{curl} A\right) \\
& +\left\|\nabla_{A}^{2} \psi\right\|_{L^{2}(\Omega)}^{2}+\int_{\partial \Omega}\left\{\sum_{j, k}\left[\overline{\nabla_{A} k \psi_{j}} \psi_{k} \nu_{j}\right]-\overline{\nabla_{A}^{2} \psi}\left(\nabla_{A} \psi\right) \cdot \nu\right\} d s \\
& =\left(|\psi|^{2} \operatorname{curl} A+2 \mathfrak{F}\left(\partial_{2} \psi \partial_{1} \bar{\psi}\right)+2 \mathfrak{R}\left(A^{1} \bar{\psi} \partial_{2} \psi-A^{2} \psi \partial_{1} \bar{\psi}\right), \operatorname{curl} A\right) \\
& +\left\|\nabla_{A}^{2} \psi\right\|_{L^{2}(\Omega)}^{2}+\int_{\partial \Omega}\left\{\sum_{j, k}\left[\overline{\nabla_{A^{k}} \psi_{j}} \psi_{k} \nu_{j}\right]-\overline{\nabla_{A}^{2} \psi}\left(\nabla_{A} \psi\right) \cdot \nu\right\} d s \\
& =\|\psi \operatorname{curl} A\|_{L^{2}(\Omega)}^{2}+\left\|\nabla_{A}^{2} \psi\right\|_{L^{2}(\Omega)}^{2}+2 \mathfrak{F}\left(\partial_{2} \psi \partial_{1} \bar{\psi}, \operatorname{curl} A\right) \\
& +2 \mathfrak{R}\left(A^{1} \bar{\psi} \partial_{2} \psi-A^{2} \psi \partial_{1} \bar{\psi}, \operatorname{curl} A\right) \\
& +\int_{\partial \Omega}\left\{\sum_{j, k}\left[\overline{\nabla_{A} k \psi_{j}} \psi_{k} \nu_{j}\right]-\overline{\nabla_{A}^{2} \psi}\left(\nabla_{A} \psi\right) \cdot \nu\right\} d s,
\end{aligned}
$$

which gives (4.3). (4.3) implies that $I(\partial \Omega)$ is real and

$$
I(\partial \Omega)=\Re \int_{\partial \Omega}\left\{\sum_{j, k}\left[\overline{F_{j, k}}+\overline{\nabla_{A^{j}} \psi_{k}}\right] \psi_{k} \nu_{j}-\overline{\nabla_{A}^{2} \psi}\left(\nabla_{A} \psi\right) \cdot \nu\right\} d s
$$

A computation shows

$$
\begin{aligned}
\Re \int_{\partial \Omega} \sum_{j, k} \overline{F_{j, k}} \psi_{k} \nu_{j} & =\Re \int_{\partial \Omega} \overline{F_{1,2}}\left(\nabla_{A^{1}} \psi \nu_{2}-\nabla_{A^{2}} \psi \nu_{1}\right) d s \\
& \left.=\Re \int_{\partial \Omega} i \overline{\psi(} \operatorname{curl} A\right)\left(\nabla_{A^{1}} \psi \nu_{2}-\nabla_{A^{2}} \psi \nu_{1}\right) d s \\
& =\Re \int_{\partial \Omega} i \bar{\psi}(\operatorname{curl} A)\left(\partial_{1} \psi \nu_{2}-\partial_{2} \psi \nu_{1}\right) d s+\int_{\partial \Omega}|\psi|^{2}(\operatorname{curl} A)\left(A^{1} \nu_{2}-A^{2} \nu_{1}\right) d s \\
& =\int_{\partial \Omega}(\operatorname{curl} A)\left[\mathfrak{F}(\bar{\psi} \nabla \psi)-|\psi|^{2} A\right] \cdot \tau d s ;
\end{aligned}
$$

and

$$
\begin{aligned}
\Re \int_{\partial \Omega} \sum_{j, k}\left[\overline{\nabla_{A^{j}} \psi_{k}}\right] \psi_{k} \nu_{j} d s & =\Re \int_{\partial \Omega} \sum_{j, k}\left(\partial_{j}+i A^{j}\right) \overline{\nabla_{A^{k}}} \nabla_{A^{k}} \psi \nu_{j} d s \\
& =\Re \int_{\partial \Omega} \sum_{j, k} \partial_{j} \overline{\nabla_{A^{k}}} \nabla_{A^{k}} \psi \nu_{j} d s=\frac{1}{2} \int_{\partial \Omega} \frac{\partial}{\partial \nu}\left|\nabla_{A} \psi\right|^{2} d s .
\end{aligned}
$$

So (4.4) is true. 
Proof of Theorem 4.1: For a given compact subset $K$ of $\Omega$, we choose a smooth real cutoff function $\eta$ such that spt $\eta \subset \Omega$ and $\eta=1$ on $K$. Denote $\psi_{j}=\nabla_{A^{j}} \psi$. From (4.3) and the Kato's inequality it follows that

$$
\begin{aligned}
\left\|\nabla\left|\nabla_{A}(\eta \psi)\right|\right\|_{L^{2}(\Omega)}^{2} \leqslant & \sum_{j, k}\left\|\nabla_{A^{j}} \nabla_{A^{k}}(\eta \psi)\right\|_{L^{2}(\Omega)}^{2} \\
= & \|\eta \psi \operatorname{curl} A\|_{L^{2}(\Omega)}^{2}+\left\|\nabla_{A}^{2}(\eta \psi)\right\|_{L^{2}(\Omega)}^{2}+2 \mathfrak{F}\left(\overline{\nabla_{A^{1}}(\eta \psi)} \nabla_{A^{2}}(\eta \psi), \operatorname{curl} A\right) \\
\leqslant & \|\eta \psi \operatorname{curl} A\|_{L^{2}(\Omega)}^{2}+\left\|\eta \nabla_{A}^{2} \psi+2 \nabla \eta \cdot \nabla_{A} \psi+\psi \Delta \eta\right\|_{L^{2}(\Omega)}^{2} \\
& +2 \int_{\Omega}\left|\operatorname{curl} A \| \nabla_{A}(\eta \psi)\right|^{2} d x
\end{aligned}
$$

Next, we estimate

$$
\begin{aligned}
\int_{\Omega}\left|\operatorname{curl} A \| \nabla_{A}(\eta \psi)\right|^{2} d x & \leqslant\|\operatorname{curl} A\|_{L^{2}(\Omega)}\left\{\int_{\Omega}\left|\nabla_{A}(\eta \psi) \| \nabla_{A}(\eta \psi)\right|^{3} d x\right\}^{1 / 2} \\
& \leqslant\|\operatorname{curl} A\|_{L^{2}(\Omega)}\left\|\nabla_{A}(\eta \psi)\right\|_{L^{2}(\Omega)}^{1 / 2}\left\|\nabla_{A}(\eta \psi)\right\|_{L^{6}(\Omega)}^{3 / 2} \\
& \leqslant C_{1}\|\operatorname{curl} A\|_{L^{2}(\Omega)}\left\|\nabla_{A}(\eta \psi)\right\|_{L^{2}(\Omega)}^{1 / 2}\left\|\nabla \mid \nabla_{A}(\eta \psi)\right\| \|_{L^{2}(\Omega)}^{3 / 2}
\end{aligned}
$$

(by Sobolev inequality)

$$
\leqslant \frac{1}{2}\left\|\nabla\left|\nabla_{A}(\eta \psi)\right|\right\|_{L^{2}(\Omega)}^{2}+C_{2}\|\operatorname{curl} A\|_{L^{2}(\Omega)}^{4}\left\|\nabla_{A}(\eta \psi)\right\|_{L^{2}(\Omega)}^{2} .
$$

Therefore,

$$
\begin{gathered}
\frac{1}{2}\left\|\nabla\left|\nabla_{A}(\eta \psi)\right|\right\|_{L^{2}(\Omega)}^{2} \leqslant \\
+\eta \psi \operatorname{curl} A\left\|_{L^{2}(\Omega)}^{2}+3\right\| \eta \nabla_{A}^{2} \psi\left\|_{L^{2}(\Omega)}^{2}+12\right\| \nabla \eta \cdot \nabla_{A} \psi \|_{L^{2}(\Omega)}^{2} \\
+3\|\psi \Delta \eta\|_{L^{2}(\Omega)}^{2}+C_{2}\|\operatorname{curl} A\|_{L^{2}(\Omega)}^{4}\left\|\nabla_{A}(\eta \psi)\right\|_{L^{2}(\Omega)}^{2} \\
\leqslant\|\psi \operatorname{curl} A\|_{L^{2}(\Omega)}^{2}+3\|g\|_{L^{2}(\Omega)}^{2}+C_{3}\left[\left\|\nabla_{A} \psi\right\|_{L^{2}(\Omega)}^{2}+\|\psi\|_{L^{2}(\Omega)}^{2}\right] \\
+C_{2}\|\operatorname{curl} A\|_{L^{2}(\Omega)}^{4}\left\|\nabla_{A}(\eta \psi)\right\|_{L^{2}(\Omega)}^{2},
\end{gathered}
$$

where $C_{1}, C_{2}, C_{3}$ are constants depending only on $\Omega$ and $K$. The proof is complete.

\section{ESTIMATES NEAR BOUNDARIES}

In this section we establish the boundary estimates for the solutions of the equation

$$
\begin{gathered}
-\nabla_{A}^{2} \psi=g \quad \text { in } \Omega, \\
\left(\nabla_{A} \psi\right) \cdot \nu+\gamma \psi=0 \quad \text { on } \partial \Omega .
\end{gathered}
$$

As mentioned in Sec. I, by making a gauge transformation if necessary, we may assume that

$$
\operatorname{div} A=0 \quad \text { in } \Omega, \quad A \cdot \nu=0 \quad \text { on } \partial \Omega .
$$

Of course, under the gauge transformation, function $g$ in (5.1) will be changed to a new function $\tilde{g}$. However, since it does not effect the estimation given below, we still denote the new function $\tilde{g}$ by $g$.

To obtain the estimates we shall straighten a portion of boundary and study the new equation in the half ball $B_{R}^{+}$. We also need to extend the solutions to the entire ball. For this purpose we 
transform Eq. (5.1) to an equation having homogeneous boundary condition. Let $u$ be the positive eigenfunction associated with the first eigenvalue $\lambda$ of the following eigenvalue problem:

$$
\begin{gathered}
-\Delta u=\lambda u \quad \text { in } \Omega, \\
\frac{\partial u}{\partial \nu}+\gamma u=0 \quad \text { on } \partial \Omega .
\end{gathered}
$$

$u$ is smooth and positive on $\bar{\Omega}$. Set $\psi=u \phi, v=\log \left(u^{2}\right), f=g / u$. Then, $\phi$ satisfies the equation

$$
\begin{gathered}
-\nabla_{A}^{2} \phi=\nabla v \cdot \nabla_{A} \phi-\lambda \phi+f \quad \text { in } \Omega, \\
\frac{\partial \phi}{\partial \nu}=0 \quad \text { on } \partial \Omega .
\end{gathered}
$$

In the following we denote $\widetilde{\phi}(y)=\phi(\mathcal{F}(y)), \widetilde{v}(y)=v(\mathcal{F}(y))$, where $\mathcal{F}(y)$ is the diffeomorphism defined on $B_{R_{0}}$, see (3.4). We shall always assume $R<R_{0} / 2$. Let $\mathbf{a}(y)$ be the vector field associated with $A(x)$ defined by (3.7). We define the following differential operators:

$$
\begin{gathered}
D(g) w=D(g)_{1} w \mathbf{e}_{1}+D(g)_{2} w \mathbf{e}_{2}, \\
\text { where } D(g)_{1}=\frac{1}{g} \partial_{1}, \quad D(g)_{2}=\partial_{2}, \\
D(g)_{\mathbf{a}} w=\left[D(g)_{\mathbf{a}^{1}} w\right] \mathbf{e}_{1}+\left[D(g)_{\mathbf{a}^{2} w} w \mathbf{e}_{2},\right. \\
\text { where } D(g)_{\mathbf{a}^{1} w} w \frac{1}{g}\left(\partial_{1}-i \mathbf{a}^{1}\right) w, \quad D(g)_{\mathbf{a}^{2} w}=\left(\partial_{2}-i \mathbf{a}^{2}\right) w, \\
D(g)_{\mathbf{a}}^{*} w=\left[D(g)_{\mathbf{a}^{1}}^{*} w\right] \mathbf{e}_{1}+\left[D(g)_{\mathbf{a}^{2}}^{*} w\right] \mathbf{e}_{2}, \\
\text { where } D(g)_{\mathbf{a}^{1}}^{*} w=D(g)_{\mathbf{a}^{1}} w, \quad D(g)_{\mathbf{a}^{2}}^{*} w=\frac{1}{g}\left[\partial_{2}(g w)-i \mathbf{a}^{2} g w\right], \\
\Delta(g)_{\mathbf{a}} w=D(g)_{\mathbf{a}^{1}}^{*} D(g)_{\mathbf{a}^{1} w+D(g)}^{*} D(g)_{\mathbf{a}^{2} w} \\
=\frac{1}{g}\left\{\partial_{1}\left[\frac{1}{g}\left(\partial_{1} w-i \mathbf{a}^{1} w\right)\right]-\frac{i \mathbf{a}^{1}}{g}\left(\partial_{1} w-i \mathbf{a}^{1} w\right)\right\} \\
\quad+\frac{1}{g}\left\{\partial_{2}\left[g\left(\partial_{2} w-i \mathbf{a}^{2} w\right)\right]-i \mathbf{a}^{2} g\left(\partial_{2} w-i \mathbf{a}^{2} w\right)\right\} .
\end{gathered}
$$

As in Sec. III we denote $\nabla_{y} \chi=\left(\partial_{1} \chi\right) \mathbf{e}_{1}+\left(\partial_{2} \chi\right) \mathbf{e}_{2}$. The operators $D(g)_{\mathbf{a}}$ and $\Delta(g)_{\mathbf{a}}$ have the following gauge invariant properties:

$$
D(g)_{\mathbf{a}+\nabla_{y \chi}}\left(e^{i \chi} \varphi\right)=e^{i \chi} D(g)_{\mathbf{a}} \varphi, \quad \Delta(g)_{\mathbf{a}+\nabla_{y \chi}}\left(e^{i \chi} \varphi\right)=e^{i \chi} \Delta(g)_{\mathbf{a}} \varphi
$$

Note that, in the above notations, $\nabla_{A} \phi=D(g)_{\mathbf{a}} \widetilde{\phi}, \nabla_{A}^{2} \phi=\Delta(g)_{\mathbf{a}} \widetilde{\phi}$. Thus, $\widetilde{\phi}$ satisfies the equation 


$$
\begin{gathered}
-\Delta(g)_{\mathbf{a}} \bar{\phi}=D(g) \widetilde{v} \cdot D(g)_{\mathbf{a}} \tilde{\phi}-\lambda \tilde{\phi}+\tilde{f} \text { on } B_{R}^{+}, \\
\frac{\partial \widetilde{\phi}}{\partial y_{2}}=0 \quad \text { on } \Gamma_{R},
\end{gathered}
$$

where $\Gamma_{R}=\left\{\left(y_{1}, 0\right):\left|y_{1}\right|<R\right\}$.

Next, we extend the solution $\widetilde{\phi}$ of Eq. (5.4) onto the entire ball. Note that

$$
\mathbf{a}^{2}=0, \quad \partial_{2} \tilde{\phi}=0 \quad \text { when } y_{2}=0 .
$$

Hence, we can evenly extend $\mathbf{a}^{1}$ and $\widetilde{\phi}$ in $y_{2}$ and oddly extend $\mathbf{a}^{2}$ in $y_{2}$. Note that although $g(y)$ is defined on the entire ball, it is not even in $y_{2}$. Therefore, for $y_{2}<0$ we define

$$
\begin{gathered}
\tilde{\phi}\left(y_{1}, y_{2}\right)=\tilde{\phi}\left(y_{1},-y_{2}\right), \quad \widetilde{v}\left(y_{1}, y_{2}\right)=\widetilde{v}\left(y_{1},-y_{2}\right), \\
g\left(y_{1}, y_{2}\right)=g\left(y_{1},-y_{2}\right), \\
\mathbf{a}^{1}\left(y_{1}, y_{2}\right)=\mathbf{a}^{1}\left(y_{1},-y_{2}\right), \quad \mathbf{a}^{2}\left(y_{1}, y_{2}\right)=-\mathbf{a}^{2}\left(y_{1},-y_{2}\right) .
\end{gathered}
$$

After such extensions, $\widetilde{\phi} \in C^{1}\left(B_{R}\right), \mathbf{a} \in C\left(B_{R}\right)$, and $\partial_{j} \mathbf{a}^{j} \in C^{1}\left(B_{R}\right)$. We further notice that $D(g)_{\mathbf{a}^{1}} \widetilde{\phi}$ is continuous and even in $y_{2}, D(g)_{\mathbf{a}^{2}} \widetilde{\phi}$ is continuous and odd in $y_{2}$, and $\Delta(g)_{\mathbf{a}} \widetilde{\phi}$ is even in $y_{2}$. Although $\partial_{2} \widetilde{v}$ is not continuous at $y_{2}=0$, it is bounded, and $D(g)_{\mathbf{a}^{2}} \widetilde{\phi}=0$ at $y_{2}=0$. Hence, $D(g) \widetilde{v} \cdot D(g)_{\mathbf{a}} \tilde{\phi}$ is continuous.

The main result in this section is the following

Theorem 5.1: Assume that $\widetilde{\phi}$ is a solution of Eq. (5.4) and is extended as the above. Then,

$$
\begin{aligned}
\sum_{j, k}\left\|D(g)_{\mathbf{a}^{\mathbf{j}}} D(g)_{\mathbf{a}^{k}} \widetilde{\phi}\right\|_{L_{g}^{2}\left(B_{R}\right)}^{2} \leqslant & 6\|\widetilde{f}\|_{L_{g}^{2}\left(B_{R}\right)}^{2}+6 \int_{B_{R}}\left|D(g) \tilde{v} \cdot D(g)_{\mathbf{a}} \widetilde{\phi}\right|^{2} g d y+C(g, R)\|\widetilde{\phi}\|_{L_{g}^{2}\left(B_{R}\right)}^{2} \\
& +C(g, R)\left\{1+\left\|\partial_{1} \mathbf{a}^{2}-\partial_{2} \mathbf{a}^{1}\right\|_{L_{g}^{2}\left(B_{R}\right)}^{4}\right\}\left\|D(g)_{\mathbf{a}} \widetilde{\phi}\right\|_{L_{g}^{2}\left(B_{R}\right)}^{2} \\
& +C(g, R) \int_{B_{R}}\left|\partial_{1} \mathbf{a}^{2}-\partial_{2} \mathbf{a}^{1}\right|^{2}|\widetilde{\phi}|^{2} d y
\end{aligned}
$$

To prove Theorem 5.1 we need an identity, see Proposition 5.3 below. Define

$$
\begin{gathered}
G_{j k} w=D(g)_{\mathbf{a}^{j}} D(g)_{\mathbf{a}^{k} w}-D(g)_{\mathbf{a}^{k}} D(g)_{\mathbf{a}^{j}} w, \\
G_{j k}^{*}=D(g)_{\mathbf{a}^{j}}^{*} D(g)_{\mathbf{a}^{k} w}-D(g)_{\mathbf{a}^{k}} D(g)_{\mathbf{a}^{j}}^{*} w .
\end{gathered}
$$

Denote

$$
[w]_{\Gamma_{R}}=\int_{\Gamma_{R}^{+}} w d y_{1}-\int_{\Gamma_{R}^{-}} w d y_{1}=\lim _{\epsilon_{1}, \epsilon_{2} \rightarrow 0}\left\{\int_{-R}^{R}\left[w\left(y_{1}, \epsilon_{1}\right)-w\left(y_{1},-\epsilon_{2}\right)\right] d y_{1}\right\} .
$$

If $w$ is continuous in $B_{R}$, then $[w]_{\Gamma_{R}}=0$. Note that $g=1$ on $\Gamma_{R}$. We have

Lemma 5.2: Assume that $\phi$ and $\psi \in C^{1}\left(B_{R} \backslash \Gamma_{R}\right)$ and the support $\operatorname{spt}(\phi) \subset B_{R}$. Then, 


$$
\int_{B_{R}}\left(D(g)_{\mathbf{a}^{j}} \phi\right) \overline{\psi g} d y=-\int_{B_{R}} \phi \overline{D(g)_{\mathbf{a}^{j}}^{*} \psi} g d y+\left[\nu_{j} \phi \bar{\psi}\right]_{\Gamma_{R}},
$$

where $\nu_{1}=0, \nu_{2}=-1$. Moreover if $\psi \in C^{2}\left(B_{R} \backslash \Gamma_{R}\right)$, then

$$
\int_{B_{R}}\left(D(g)_{\mathbf{a}^{j}} \phi\right) \overline{D(g)_{\mathbf{a}^{k}} \psi} g d y=-\int_{B_{R}} \phi \overline{D(g)_{\mathbf{a}^{j}}^{*} D(g)_{\mathbf{a}^{k}} \psi} g d y+\left[\nu_{j} \phi \overline{D(g)_{\mathbf{a}^{k}} \psi}\right]_{\Gamma_{R}} .
$$

For our convenience we denote by $\|\cdot\|_{L_{g}^{2}}$ the $L^{2}$ norm with the weight $g$, and $(\phi, w)_{g}$ $=\int_{B_{R}} \phi \overline{w g} d y$.

Proposition 5.3: Assume that $\psi \in C^{1}\left(B_{R}\right) \cap C^{2}\left(B_{R} \backslash \Gamma_{R}\right)$ with its support $\operatorname{spt}(\psi) \subset B_{R}$, and $\psi$ is even in $y_{2}$. Then,

$$
\begin{aligned}
\sum_{j, k}\left\|D(g)_{\mathbf{a}^{j}} D(g)_{\mathbf{a}^{k}} \psi\right\|_{L_{g}^{2}\left(B_{R}\right)}^{2}= & \left\|\Delta(g)_{\mathbf{a}} \psi\right\|_{L_{g}^{2}\left(B_{R}\right)}^{2}+\left\|G_{12} \psi\right\|_{L_{g}^{2}\left(B_{R}\right)}^{2}+\int_{B_{R}}\left|D(g)_{\mathbf{a}^{2}} \psi\right|^{2} g \partial_{2}\left(\frac{\partial_{2} g}{g}\right) d y \\
& -2 \mathfrak{F} \int_{B_{R}}\left(\partial_{1} \mathbf{a}^{2}-\partial_{2} \mathbf{a}^{1}\right)\left(D(g)_{\mathbf{a}^{1} \psi}\right) \overline{D(g)_{\mathbf{a}^{2}} \psi} d y-2 \mathfrak{R} \int_{B_{R}}\left(\partial_{2} g\right) \\
& \times\left(D(g)_{\mathbf{a}^{2}} \psi\right) \overline{D(g)_{\mathbf{a}^{1}}^{2} \psi} d y+\int_{B_{R}}\left[\partial_{1}\left(\frac{\partial_{2} g}{g}\right)-\frac{\partial_{1} g \partial_{2} g}{g}\right] \\
& \times\left(D(g)_{\mathbf{a}^{1} \psi} \psi \overline{D(g)_{\mathbf{a}^{2}} \psi} d y .\right.
\end{aligned}
$$

Remark 5.1: Note that curl $\mathbf{a}=\left(\partial_{1} \mathbf{a}^{2}-\partial_{2} \mathbf{a}^{1}\right) / g$. Although it is not continuous at $y_{2}=0$, it remains bounded. The term $\partial_{1}\left(\left(\partial_{2} g\right) / g\right)-\left(\partial_{1} g\right)\left(\partial_{2} g\right) / g$ is also bounded. Therefore, the integrals involving such terms make sense. Also note that when $y_{2} \neq 0, g \partial_{2}\left(\left(\partial_{2} g\right) / g\right)=-\left[\kappa_{r}\left(y_{1}\right)\right]^{2} / g$ $\leqslant 0$. So

$$
\int_{B_{R}}\left|D(g)_{\mathbf{a}^{2}} \psi\right|^{2} g \partial_{2}\left(\frac{\partial_{2} g}{g}\right) d y \leqslant 0
$$

Proof of Proposition 5.3: The proof is similar to one of Proposition 4.3, but involves more computations. Set $\psi_{j}=D(g)_{\mathbf{a}^{j}} \psi$. Using Lemma 5.2 we have

$$
\begin{aligned}
\left(D(g)_{\mathbf{a}^{j}} D(g)_{\mathbf{a}^{k}} \psi, D(g)_{\mathbf{a}^{j}} D(g)_{\mathbf{a}^{k}} \psi\right)_{g} \\
=\left(D(g)_{\mathbf{a}^{j}} \psi_{k}, G_{j k} \psi+D(g)_{\mathbf{a}^{k}} \psi_{j}\right)_{g} \\
=\left(D(g)_{\mathbf{a}^{j}} \psi_{k}, G_{j k} \psi\right)_{g}+\left(D(g)_{\mathbf{a}^{j}} \psi_{k}, D(g)_{\mathbf{a}^{k}} \psi_{j}\right)_{g} \\
=\left(D(g)_{\mathbf{a}^{j}} \psi_{k}, G_{j k} \psi\right)_{g}-\left(\psi_{k}, G_{j k}^{*} \psi_{j}\right)_{g}+\left(D(g)_{\mathbf{a}^{k}}^{*} \psi_{k}, D(g)_{\mathbf{a}^{j}}^{*} \psi_{j}\right)_{g} \\
\quad+\left[\nu_{j} \psi_{k} \overline{D(g)_{\mathbf{a}^{k}} \psi_{j}}\right]_{\Gamma_{R}}-\left[\nu_{k} \psi_{k} \overline{D(g)_{\mathbf{a}^{j}}^{*} \psi_{j}}\right]_{\Gamma_{R}} .
\end{aligned}
$$

Summing up the above over $1 \leqslant j, k \leqslant 2$ we have

$$
\begin{aligned}
\sum_{j, k}\left\|D(g)_{\mathbf{a}^{j}} D(g)_{\mathbf{a}^{k}} \psi\right\|_{L_{g^{2}\left(B_{R}\right)}^{2}=}^{2} & \left\|\Delta(g)_{\mathbf{a}} \psi\right\|_{L_{g}^{2}\left(B_{R}\right)}^{2}+\sum_{j, k}\left\{\left(D(g)_{\mathbf{a}^{j}} \psi_{k}, G_{j k} \psi\right)_{g}-\left(\psi_{k}, G_{j k}^{*} \psi_{j}\right)_{g}\right\} \\
& +\sum_{j k}\left\{\left[\nu_{j} \psi_{k} \overline{D(g)_{\mathbf{a}^{k}} \psi_{j}}\right]_{\Gamma_{R}}-\left[\nu_{k} \psi_{k} \overline{D(g)_{\mathbf{a}^{j}}^{*} \psi_{j}}\right]_{\Gamma_{R}}\right\} .
\end{aligned}
$$


Since $\nu_{1}=0, \nu_{2}=-1, \psi_{2}=D(g)_{\mathbf{a}^{2}} \psi=0$ on $\Gamma_{R}$, we have $\nu_{k} \psi_{k}=0$ on $\Gamma_{R}$, and

$$
\sum_{j k}\left\{\left[\nu_{j} \psi_{k} \overline{D(g)_{\mathbf{a}^{k}} \psi_{j}}\right]_{\Gamma_{R}}-\left[\nu_{k} \psi_{k} \overline{D(g)_{\mathbf{a}}^{*} \psi_{j}}\right]_{\Gamma_{R}}\right\}=-\left[\left(D(g)_{\mathbf{a}} 1 \psi\right) \overline{D(g)_{\mathbf{a}^{1}} \psi_{2}}\right]_{\Gamma_{R}}=0
$$

Here the following fact is used:

$$
D(g)_{\mathbf{a}^{1}} \psi_{2}=D(g)_{\mathbf{a}^{1}} D(g)_{\mathbf{a}^{2}} \psi=\frac{1}{g}\left[\partial_{1} \partial_{2} \psi-i \mathbf{a}^{1} \partial_{2} \psi-i \partial_{1}\left(\mathbf{a}^{2} \psi\right)-\mathbf{a}^{1} \mathbf{a}^{2} \psi\right] \rightarrow 0 \quad \text { as } \quad y_{2} \rightarrow 0
$$

Therefore,

$$
\begin{aligned}
\sum_{j, k} & \left\|D(g)_{\mathbf{a}^{j}} D(g)_{\mathbf{a}^{k}} \psi\right\|_{L_{g}^{2}\left(B_{R}\right)}^{2} \\
& =\left\|\Delta(g)_{\mathbf{a}} \psi\right\|_{L_{g}^{2}\left(B_{R}\right)}^{2}+\left(D(g)_{\mathbf{a}^{1}} \psi_{2}-D(g)_{\mathbf{a}^{2}} \psi_{1}, G_{12} \psi\right)_{g}-\sum_{j k}\left(\psi_{k}, G_{j k}^{*} \psi_{j}\right)_{g} \\
& =\left\|\Delta(g)_{\mathbf{a}} \psi\right\|_{L_{g}^{2}\left(B_{R}\right)}^{2}+\left\|G_{12} \psi\right\|_{L_{g}^{2}\left(B_{R}\right)}^{2}-\sum_{j k}\left(\psi_{k}, G_{j k} \psi_{j}\right)_{g}+\sum_{k}\left(\psi_{k}, \psi_{2} D(g)_{k}\left[\frac{\partial_{2} g}{g}\right]\right)_{g}
\end{aligned}
$$

By computation we get

$$
\begin{aligned}
& \sum_{k}\left(\psi_{k}, \psi_{2} D(g)_{k}\left[\frac{\partial_{2} g}{g}\right]\right)_{g}=\int_{B_{R}}\left|D(g)_{\mathbf{a}^{2}} \psi\right|^{2} g \partial_{2}\left(\frac{\partial_{2} g}{g}\right) d y+\int_{B_{R}} \partial_{1}\left(\frac{\partial_{2} g}{g}\right)\left(D(g)_{\mathbf{a}^{1}} \psi\right) \overline{D(g)_{\mathbf{a}^{2}} \psi} d y, \\
& \sum_{j k}\left(\psi_{k}, G_{j k} \psi_{j}\right)_{g}= 2 \mathfrak{F} \int_{B_{R}}\left(\partial_{1} \mathbf{a}^{2}-\partial_{2} \mathbf{a}^{1}\right)\left(D(g)_{\mathbf{a}^{1}} \psi\right) \overline{D(g)_{\mathbf{a}^{2}} \psi} d y+2 \mathfrak{R} \int_{B_{R}}\left(\partial_{2} g\right) \psi_{2} \overline{D(g)_{\mathbf{a}^{1}} \psi_{1}} d y \\
&+\int_{B_{R}} \frac{\partial_{1} g \partial_{2} g}{g}\left(D(g)_{\mathbf{a}^{1}} \psi\right) \overline{D(g)_{\mathbf{a}^{2}} \psi} d y .
\end{aligned}
$$

For instance, to obtain (5.9), we note that

$$
\begin{aligned}
\sum_{j k}\left(\psi_{k}, G_{j k} \psi_{j}\right)_{g}= & -\left(\psi_{1}, G_{12} \psi_{2}\right)_{g}+\left(\psi_{2}, G_{12} \psi_{2}\right)_{g} \\
= & \int_{B_{R}} g\left[\psi_{2} \overline{G_{12} \psi_{1}}-\psi_{1} \overline{G_{12} \psi_{2}}\right] d y \\
= & 2 \mathfrak{F} \int_{B_{R}}\left(\partial_{1} \mathbf{a}^{2}-\partial_{2} \mathbf{a}^{1}\right)\left(D(g)_{\mathbf{a}} 1 \psi\right) \overline{D(g)_{\mathbf{a}^{2}} \psi} d y \\
& +\int_{B_{R}}\left(\partial_{2} g\right)\left[\psi_{2} \overline{D(g)_{\mathbf{a}^{1}} \psi_{1}}-\psi_{1} \overline{D(g)_{\mathbf{a}^{1}} \psi_{2}}\right] d y
\end{aligned}
$$

Since 


$$
\begin{aligned}
& \int_{B_{R}}\left(\partial_{2} g\right)\left[\psi_{2} \overline{D(g)_{\mathbf{a}^{1}} \psi_{1}}-\psi_{1} \overline{D(g)_{\mathbf{a}^{1}} \psi_{2}}\right] d y \\
& \quad=\int_{B_{R}}\left(\partial_{2} g\right)\left[\psi_{2} \overline{D(g)_{\mathbf{a}^{1}} \psi_{1}}+\overline{\psi_{2}}\left(D(g)_{\mathbf{a}}{ }^{1} \psi_{1}\right)\right] d y+\int_{B_{R}} \frac{\partial_{1} g \partial_{2} g}{g} \psi_{1} \overline{\psi_{2}} d y \\
& \quad=2 \Re \int_{B_{R}}\left(\partial_{2} g\right) \psi_{2} \overline{D(g)_{\mathbf{a}^{1}} \psi_{1}}+\int_{B_{R}} \frac{\partial_{1} g \partial_{2} g}{g} \psi_{1} \overline{\psi_{2}} d y,
\end{aligned}
$$

so (5.9) holds.

Now, (5.6) follows from (5.7) to (5.9).

Proof of Theorem 5.1: In the proof, for simplicity we denote $\tilde{\phi}$ by $\phi$ and denote a constant depending only on $g$ and $R$ by $C$. Let $\eta$ be a smooth cutoff function supported in $B_{2 R}$ such that $\eta=1$ on $B_{R / 2}$ and $\eta$ is even in $y_{2}$. Using Proposition 5.3 we have

$$
\begin{aligned}
\sum_{j, k}\left\|D(g)_{\mathbf{a}^{j}} D(g)_{\mathbf{a}^{k}}(\eta \phi)\right\|_{L_{g}^{2}\left(B_{R}\right)}^{2} \leqslant & \left\|\Delta(g)_{\mathbf{a}}(\eta \phi)\right\|_{L_{g}^{2}\left(B_{R}\right)}^{2}+\left\|G_{12} \psi\right\|_{L_{g}^{2}\left(B_{R}\right)}^{2} \\
& +C\left\{\left\|D(g)_{\mathbf{a}} \phi\right\|_{L_{g}^{2}\left(B_{R}\right)}^{2}+\|\phi\|_{L_{g}^{2}\left(B_{R}\right)}^{2}\right\}+J_{1}+2 J_{2},
\end{aligned}
$$

where

$$
\begin{gathered}
J_{1}=\int_{B_{R}}\left|\partial_{1} \mathbf{a}^{2}-\partial_{2} \mathbf{a}^{1}\right|\left|D(g)_{\mathbf{a}}(\eta \phi)\right|^{2} d y, \\
J_{2}=\int_{B_{R}}\left|\partial_{2} g \| D(g)_{\mathbf{a}^{2}}(\eta \phi)\right|\left|D(g)_{\mathbf{a}^{1}}^{2}(\eta \phi)\right| d y .
\end{gathered}
$$

Now, we estimate each term on the right of (5.10),

$$
\begin{aligned}
& \left\|\Delta(g)_{\mathbf{a}}(\eta \phi)\right\|_{L_{g}^{2}\left(B_{R}\right)}^{2} \leqslant 3\|\widetilde{f}\|_{L_{g}^{2}\left(B_{R}\right)}^{2}+C\left\{\|\phi\|_{L_{g}^{2}\left(B_{R}\right)}^{2}+\left\|D(g)_{\mathbf{a}} \phi\right\|_{L_{g}^{2}\left(B_{R}\right)}^{2}\right\} \\
& +3 \int_{B_{R}}\left|D(g) \widetilde{v} \cdot D(g)_{\mathbf{a}} \phi\right|^{2} g d y, \\
& \left\|G_{12} \psi\right\|_{L_{g}^{2}\left(B_{R}\right)}^{2} \leqslant C\left\{\left\|D(g)_{\mathbf{a}} \phi\right\|_{L_{g}^{2}\left(B_{R}\right)}^{2}+\int_{B_{R}}\left|\partial_{1} \mathbf{a}^{2}-\partial_{2} \mathbf{a}^{1} \| \phi\right|^{2} d y\right\}, \\
& J_{1} \leqslant\left\|\partial_{1} \mathbf{a}^{2}-\partial_{2} \mathbf{a}^{1}\right\|_{L_{g}^{2}\left(B_{R}\right)}\left\{\int_{B_{R}}\left|D(g)_{\mathbf{a}}(\eta \phi) \| D(g)_{\mathbf{a}}(\eta \phi)\right|^{3} d y\right\}^{1 / 2} \\
& \leqslant C\left\|\partial_{1} \mathbf{a}^{2}-\partial_{2} \mathbf{a}^{1}\right\|_{L_{g}^{2}\left(B_{R}\right)}\left\|D(g)_{\mathbf{a}}(\eta \phi)\right\|_{L_{g}^{2}\left(B_{R}\right)}^{1 / 2}\left\|\nabla\left|D(g)_{\mathbf{a}}(\eta \phi)\right|\right\|_{L_{g}^{2}\left(B_{R}\right)}^{3 / 2}
\end{aligned}
$$

(by Sobolev inequality)

$$
\leqslant \epsilon\left\|\nabla\left|D(g)_{\mathbf{a}}(\eta \phi)\right|\right\|_{L_{g}^{2}\left(B_{R}\right)}^{2}+\frac{C}{\epsilon}\left\|\partial_{1} \mathbf{a}^{2}-\partial_{2} \mathbf{a}^{1}\right\|_{L_{g}^{2}\left(B_{R}\right)}^{4}\left\|D(g)_{\mathbf{a}}(\eta \phi)\right\|_{L_{g}^{2}\left(B_{R}\right)}^{2},
$$

and 


$$
\begin{aligned}
J_{2} & \leqslant \epsilon\left\|D(g)_{\mathbf{a}^{1}}^{2}(\eta \phi)\right\|_{L_{g}^{2}\left(B_{R}\right)}^{2}+\frac{C}{\boldsymbol{\epsilon}}\left\|D(g)_{\mathbf{a}}(\eta \phi)\right\|_{L_{g}^{2}\left(B_{R}\right)}^{2} \\
& \leqslant \epsilon \sum_{j k}\left\|D(g)_{\mathbf{a} j} D(g)_{\mathbf{a}^{k}}(\eta \phi)\right\|_{L_{g}^{2}\left(B_{R}\right)}^{2}+\frac{C}{\boldsymbol{\epsilon}}\left\|D(g)_{\mathbf{a}}(\eta \phi)\right\|_{L_{g}^{2}\left(B_{R}\right)}^{2} .
\end{aligned}
$$

Plugging the above inequalities back in (5.10), using the following

$$
\left\|\nabla \mid D(g)_{\mathbf{a}}(\eta \phi)\right\|_{L_{g}^{2}\left(B_{R}\right)}^{2} \leqslant C(g, R) \sum_{j k}\left\|D(g)_{\mathbf{a}^{j}} D(g)_{\mathbf{a}^{k}}(\eta \phi)\right\|_{L_{g}^{2}\left(B_{R}\right)}^{2}
$$

and choosing $\epsilon$ small enough, we obtain the estimate (5.5).

In the same fashion as the above, one can also prove the following

Lemma 5.4: Assume that $\widetilde{\phi}$ is a solution of Eq. (5.4) and is extended even in $y_{2}$. Then,

$$
\int_{B_{R / 2}}\left|D(g)_{\mathbf{a}} \widetilde{\boldsymbol{\phi}}\right|^{2} g d y \leqslant C(g, R) \int_{B_{R}}\left\{|\widetilde{\phi}|^{2}+|\widetilde{f}|^{2}+|D(g) \widetilde{v}|^{2}|\tilde{\phi}|^{2}\right\} g d y
$$

Note that after extension $\partial_{1} \mathbf{a}^{2}-\partial_{2} \mathbf{a}^{1}$ is not continuous at $\Gamma_{R}$. Therefore, the estimates depending only on the data given on $B_{R}^{+}$are needed. As a direct corollary of Theorem 5.1 we have

Theorem 5.5: Assume that $\widetilde{\phi}$ is a solution of Eq. (5.4). Then,

$$
\sum_{j k}\left\|D(g)_{\mathbf{a}^{j}} D(g)_{\mathbf{a}^{k}} \widetilde{\boldsymbol{\phi}}\right\|_{L_{g}^{2}\left(B_{R}^{+}\right)}^{2} \leqslant C\left\{\|\widetilde{f}\|_{L_{g}^{2}\left(B_{R}^{+}\right)}^{2}+\|\widetilde{\phi}\|_{\left.L_{g^{2}\left(B_{R}^{+}\right)}^{2}\right\}}\right.
$$

where the constant $C$ depends on $R, g,\|D(g) \widetilde{v}\|_{L_{g}^{2}\left(B_{R}^{+}\right)}$and $\left\|\partial_{1} \mathbf{a}^{2}-\partial_{2} \mathbf{a}^{1}\right\|_{L_{g}^{2}\left(B_{R}^{+}\right)}$.

\section{UPPER-BOUND ESTIMATES}

In this section, we give an upper bound for $\mu(\sigma A) /|\sigma|$. Throughout this section we assume $A \in C^{2}(\Omega)$.

Lemma 6.1: Assume that $A \in C^{2}(\Omega)$. Then,

$$
\limsup _{\sigma \rightarrow \infty} \frac{\mu(\sigma A)}{|\sigma|} \leqslant \min |\operatorname{curl} A(x)| .
$$

Proof: Let $H(x)=\operatorname{curl} A(x)$. First, we note that $\mu(-\sigma A)=\mu(\sigma A)$. In fact, for every $\psi$ $\in W^{1,2}(\Omega)$ we set $\phi=\bar{\psi}$. Then, $\left|\nabla_{-\sigma A} \phi\right|=\left|\overline{\nabla_{\sigma A} \psi}\right|=\left|\nabla_{\sigma A} \psi\right|$. Therefore, we may assume $\sigma>0$. We shall show that for every $x_{0} \in \Omega$,

$$
\limsup _{\sigma \rightarrow+\infty} \frac{\mu(\sigma A)}{\sigma} \leqslant\left|H\left(x_{0}\right)\right|
$$

Without loss of generality we may assume $x_{0}=0$.

Set $h=H(0)$. When $h=0$ the conclusion is obvious, see Proposition 6.3 below. So, we assume $h \neq 0$. Denote $\delta=1 / \sqrt{\sigma}$. Let $R>0$ be fixed such that $B_{R} \subset \Omega$. For any $\psi \in W^{1,2}(\Omega)$, we let $\psi_{\delta}(x)=\psi(\delta x)$ and $A_{\delta}(x)=A(\delta x) / \delta$. Then, 


$$
\begin{aligned}
\frac{\mu(\sigma A)}{\sigma} & \leqslant \frac{1}{\sigma} \inf _{\psi \in W_{0}^{1,2}\left(B_{R}\right)} \frac{\int_{B_{R}}\left|\nabla_{\sigma A} \psi\right|^{2} d x}{\int_{B_{R}}|\psi|^{2}}, \\
& =\inf _{\phi \in W_{0}^{1,2}\left(B_{R / \delta}\right)} \frac{\int_{B_{R / \delta}}\left|\nabla_{A_{\delta}} \phi\right|^{2} d x}{\int_{B_{R / \delta}}|\phi|^{2} d x} .
\end{aligned}
$$

Using (3.1) and noting that $\nabla \xi(\delta x)=\delta \nabla \xi(x), \omega(\delta x)=\delta \omega(x)$, we have

$$
A_{\delta}(x)=\nabla \chi_{\delta}(x)+h \omega(x)+B_{\delta}(x),
$$

where

$$
\begin{gathered}
\chi_{\delta}(x)=\frac{1}{\delta} A(0) \cdot x+\xi(x)+\delta \zeta(x), \\
B_{\delta}(x)=-\frac{\delta}{2}|x|^{2} \operatorname{curl}^{2} A(0)+\frac{1}{\delta} D(\delta x), \\
\left|B_{\delta}(x)\right| \leqslant \frac{\delta}{2}\left|\operatorname{curl}^{2} A(0)\right||x|^{2}[1+o(\delta R)] \quad \text { in } B_{R / \delta} .
\end{gathered}
$$

Therefore,

$$
\left|\nabla_{A_{\delta}} e^{i \chi_{\delta}} \phi\right|^{2}=\left|\nabla_{h \omega+B_{\delta}} \phi\right|^{2}=\left|\nabla_{h \omega} \phi-i B_{\delta} \phi\right|^{2} \leqslant(1+\lambda)\left|\nabla_{h \omega} \phi\right|^{2}+\frac{(1+\lambda) \delta^{2}}{4 \lambda}(1+o(\delta R))|x|^{4}|\phi|^{2},
$$

where $0 \leqslant \lambda \leqslant 1$. So,

$$
\begin{aligned}
\frac{\mu(\sigma A)}{\sigma} \leqslant & \inf _{\phi \in W_{0}^{1,2}\left(B_{R / \delta}\right)} \frac{1}{\int_{B_{R / \delta}}|\phi|^{2} d x}\left\{(1+\lambda) \int_{R_{R / \delta}}\left|\nabla_{h \omega} \phi\right|^{2} d x\right. \\
& \left.+\frac{(1+\lambda) \delta^{2}}{4 \lambda}(1+o(\delta R))\left|\operatorname{curl}^{2} A(0)\right|^{2} \int_{R_{R / \delta}}|x|^{4}|\phi|^{2} d x\right\} .
\end{aligned}
$$

Choose $\phi=\phi_{m}=u \eta_{m}$, where $u(x)=u(|x|)=\exp \left(-h^{2}|x|^{2} / 4\right)$ and $\eta_{m}$ is a smooth cutoff function supported in $B_{m}$ such that $\eta_{m} \equiv 1$ on $B_{m / 2}$. For fixed $R$ and for all small $\delta$,

$$
\begin{aligned}
\frac{\mu(\sigma A)}{\sigma} \leqslant & \frac{1}{\int_{B_{m}}\left|\phi_{m}\right|^{2} d x}\left\{(1+\lambda) \int_{R_{m}}\left|\nabla_{h \omega} \phi_{m}\right|^{2} d x\right. \\
& \left.+\frac{(1+\lambda) \delta^{2}}{4 \lambda}(1+o(\delta R))\left|\operatorname{curl}^{2} A(0)\right|^{2} \int_{R_{m}}|x|^{4}\left|\phi_{m}\right|^{2} d x\right\} .
\end{aligned}
$$

We first fix $m>1, \lambda \in(0,1)$ and let $\sigma$ approach $+\infty$ (so $\delta \rightarrow 0$ ), then we fix $m$ and send $\lambda$ to 0 , finally we send $m$ to $+\infty$. By using Lemma 2.1, we obtain

$$
\limsup _{\sigma \rightarrow+\infty} \frac{\mu(\sigma A)}{\sigma} \leqslant \frac{\int_{\mathbb{R}^{2}}\left|\nabla_{h \omega} u\right|^{2} d x}{\int_{\mathbb{R}^{2}}|u|^{2} d x}=\alpha(h)=|h|=|H(0)| .
$$

This completes the proof.

Lemma 6.2: Assume that $A \in C^{2}(\bar{\Omega})$. Then, 


$$
\limsup _{\sigma \rightarrow \infty} \frac{\mu(\sigma A)}{|\sigma|} \leqslant \beta_{0} \min _{x \in \partial \Omega}|\operatorname{curl} A(x)|
$$

where $\beta_{0}$ is given in Lemma 2.2 .

Proof: As in the proof of Lemma 6.1, we only need to show that, if $0 \in \partial \Omega$ and $h=H(0)$, then

$$
\limsup _{\sigma \rightarrow+\infty} \frac{\mu(\sigma A)}{|\sigma|} \leqslant \beta_{0}|h|
$$

Now, we need to use the local decomposition of $A$ in the new variables which straighten a portion of boundary of $\partial \Omega$ near the point 0 . We shall use the notations presented in Sec. III. For a fixed small $R>0$, we have

$$
\mu(\sigma A) \leqslant \inf _{\phi \in W^{*}\left(B_{R}^{+}\right)} \frac{\int_{B_{R}^{+}}\left|D(g)_{\sigma \mathbf{a}} \phi\right|^{2} g(y) d y+\gamma \int_{-R}^{R}|\phi|^{2} d y_{1}}{\int_{B_{R}^{+}}|\phi|^{2} g(y) d y},
$$

where $W^{*}\left(B_{R}^{+}\right)=\left\{\phi \in W^{1,2}\left(B_{R}^{+}\right): \operatorname{spt}(\phi) \subset \overline{B_{R}}\right\}$, a is the vector field associated with $A$ given by (3.7).

For $\sigma>0$ we set $\delta=1 / \sqrt{\sigma}, \mathbf{a}_{\delta}(y)=(1 / \delta) \mathbf{a}(\delta y), g_{\delta}(y)=g(\delta y)$. Then, for all small $\delta$,

$$
\frac{\mu(\sigma A)}{\sigma} \leqslant \inf _{\phi \in W^{*}\left(B_{R / \delta}^{+}\right)} \frac{\int_{B_{R / \delta}^{+}\left|D\left(g_{\delta}\right)_{\mathbf{a}_{\delta}} \phi\right|^{2} g_{\delta}(y) d y+\gamma \delta \int_{-R / \delta}^{R / \delta}|\phi|^{2} d y_{1}}}{\int_{B_{R / \delta}^{+}|\phi|^{2} g_{\delta}(y) d y}} .
$$

From (3.11) we have

$$
\mathbf{a}_{\delta}(y)=\nabla_{y} \tilde{\chi}_{\delta}(y)+h \tilde{\omega}(y)+\widetilde{B}_{\delta}(y),
$$

where

$$
\begin{gathered}
\tilde{\chi}_{\delta}(y)=\frac{1}{\delta} A(0) \cdot y+\widetilde{\xi}(y)+\delta \widetilde{\zeta}(y), \\
\widetilde{\omega}(y)=\left(-y_{2} / 2\right) \mathbf{e}_{1}+\left(y_{1} / 2\right) \mathbf{e}_{2}, \\
\widetilde{B}_{\delta}(y)=-\frac{\delta|y|^{2}}{2}\left[\operatorname{curl}^{2} A(0)-h \kappa_{r}(0) \tau(0)\right]+\frac{1}{\delta} \widetilde{D}(\delta y) .
\end{gathered}
$$

Here $\kappa_{r}(0)$ is the relative curvature of $\partial \Omega$ at the point 0 . Since the operator $D(g)_{\mathbf{a}}$ is gauge invariant, see (5.3), so

$$
\left|D\left(g_{\delta}\right)_{\mathbf{a}_{\delta}} \exp \left(i \widetilde{\chi}_{\delta}\right) \phi\right|^{2}=\left|D\left(g_{\delta}\right)_{h \omega+\tilde{B}_{\delta}} \phi\right|^{2} \leqslant(1+\lambda)\left|D\left(g_{\delta}\right)_{h \omega} \phi\right|^{2}+\frac{1+\lambda}{\lambda\left|g_{\delta}\right|^{2}}\left|\widetilde{B}_{\delta} \phi\right|^{2}
$$

where $\lambda$ is an arbitrary number lying between 0 and 1 .

Choose $\phi=\psi \eta_{m}$, where $\psi \in \mathcal{W}\left(\mathbb{R}_{+}^{2}\right), \eta_{m}$ is the cutoff function used in the proof of Lemma 6.1. Note that $g_{\delta} \rightarrow 1$ uniformly on each $B_{m}^{+}$as $\delta \rightarrow 0$. Therefore, by the same argument as in the proof of Lemma 6.1, we obtain

$$
\limsup _{\sigma \rightarrow+\infty} \frac{\mu(\sigma A)}{\sigma} \leqslant \frac{\int_{\mathbb{R}_{+}^{2}}\left|\nabla_{h \omega} \psi\right|^{2} d y}{\int_{R_{+}^{2}}|\psi|^{2} d y} .
$$


Since the above is true for all $\psi \in \mathcal{W}\left(R_{+}^{2}\right)$, using Lemma 2.2 we have

$$
\limsup _{\sigma \rightarrow+\infty} \frac{\mu(\sigma A)}{\sigma} \leqslant \beta(h)=\beta_{0}|h|=\beta_{0}|H(0)|
$$

The proof is complete.

If $\operatorname{curl} A$ vanishes at some point in $\bar{\Omega}$, the estimates (6.1), (6.2) can be greatly improved. Denote

$$
\mathcal{Z}(A, \Omega)=\{x \in \Omega: \operatorname{curl} A(x)=0\}, \quad \mathcal{Z}(A, \partial \Omega)=\{x \in \partial \Omega: \operatorname{curl} A(x)=0\} .
$$

Define, for $\tau>0$,

$$
p(\tau)=\inf _{u \in \mathcal{W}\left(\mathbb{R}^{2}\right)} \frac{\int_{\mathbb{R}^{2}}\left\{|\nabla u|^{2}+\frac{1}{4} \tau^{2}|x|^{4}|u|^{2}\right\} d x}{\int_{\mathbb{R}^{2}}|u|^{2} d x} .
$$

Using the rescaling method we can show that $p(\tau)=p(1)|\tau|^{2 / 3}$ and $p(\tau)$ is achieved for every $\tau \neq 0$. Choosing $u=\exp \left(-|x|^{3} / 6\right)$ as a test function we see that $p(1) \leqslant \sqrt[3]{3} / \Gamma\left(\frac{5}{3}\right)$. Define, for a constant vector $\mathbf{a}$,

$$
q(\mathbf{a})=\inf _{\phi \in \mathcal{W}\left(\mathbb{R}_{+}^{2}\right)} \frac{\left.\left.\int_{\mathbb{R}_{+}^{2}}\left|\nabla \phi-\frac{i}{2}\right| y\right|^{2} \mathbf{a} \phi\right|^{2} d y}{\int_{\mathbb{R}_{+}^{2}}|\phi|^{2} d y} .
$$

Obviously,

$$
q(\mathbf{a}) \leqslant \inf _{\phi \in \mathcal{W}\left(\mathbb{R}_{+}^{2}\right)} \frac{\int_{\mathbb{R}_{+}^{2}}\left\{|\nabla \phi|^{2}+\frac{1}{4}|\mathbf{a}|^{2}|y|^{2}|\phi|^{2}\right\} d y}{\int_{\mathbb{R}_{+}^{2}}|\phi|^{2} d y} \leqslant p(|\mathbf{a}|)=p(1)|\mathbf{a}|^{2 / 3} \leqslant \frac{\sqrt[3]{3}}{\Gamma\left(\frac{5}{3}\right)}|\mathbf{a}|^{2 / 3}
$$

Proposition 6.3: Assume that $A \in C^{2}(\bar{\Omega})$. If $\mathcal{Z}(A, \Omega) \neq \varnothing$, then

$$
\limsup _{\sigma \rightarrow \infty} \frac{\mu(\sigma A)}{|\sigma|^{2 / 3}} \leqslant p(1) \inf _{x \in \mathcal{Z}(A, \Omega)}\left|\operatorname{curl}^{2} A(x)\right|^{2 / 3} .
$$

If $\mathcal{Z}(A, \partial \Omega) \neq \varnothing$, then

$$
\limsup _{\sigma \rightarrow \infty} \frac{\mu(\sigma A)}{|\sigma|^{2 / 3}} \leqslant \inf _{x \in \mathcal{Z}(A, \partial \Omega)} q\left(\operatorname{curl}^{2} A(x)\right) \leqslant p(1) \inf _{x \in \mathcal{Z}(A, \partial \Omega)}\left|\operatorname{curl}^{2} A(x)\right|^{2 / 3}
$$

Here $p(1)$ and $q(\mathbf{a})$ are defined in (6.3), (6.4).

Remark 6.1: Note that $\left|\operatorname{curl}^{2} A(x)\right|=|\nabla H(x)|$, where $H(x)=\operatorname{curl} A(x)$.

Proof of Proposition 6.3: Assume that $0 \in \mathcal{Z}(A, \Omega)$. For $\sigma>0$ we set $\delta=1 / \sqrt[3]{\sigma}, \psi_{\delta}(x)$ $=\psi(\delta x), A_{\delta}(x)=A(\delta x) / \delta^{2}$. From Lemma 3.1, we have

$$
A_{\delta}(x)=\nabla \hat{\chi}_{\delta}(x)-\frac{1}{2}|x|^{2} \operatorname{curl}^{2} A(0)+D_{\delta}(x),
$$

where 


$$
\begin{gathered}
\hat{\chi}_{\delta}(x)=\frac{1}{\delta^{2}} A(0) \cdot x+\frac{1}{\delta} \xi(x)+\zeta(x) \\
D_{\delta}(x)=\frac{1}{\delta^{2}} D(\delta x), \quad\left|D_{\delta}(x)\right|=o\left(\delta|x|^{3}\right) \quad \text { in } B_{R / \delta} .
\end{gathered}
$$

Set $\phi_{m}=u \eta_{m}$, where $\eta_{m}$ is a smooth cutoff function as we used above and $u$ is a real function to be determined later. Then, we have

$$
\begin{aligned}
\frac{\mu(\sigma A)}{\sigma^{2 / 3}} \leqslant & \frac{1}{\int_{B_{m}}\left|\phi_{m}\right|^{2} d x}\left\{(1+\lambda) \int_{R_{m}}\left[\left|\nabla \phi_{m}\right|^{2}+\frac{1}{4}\left|\operatorname{curl}^{2} A(0)\right|^{2}|x|^{4}\left|\phi_{m}\right|^{2}\right] d x\right. \\
& \left.+O(\delta)\left(1+\frac{1}{\lambda}\right) \int_{R_{m}}|x|^{6}\left|\phi_{m}\right|^{2} d x\right\} .
\end{aligned}
$$

First sending $\delta$ to 0 , then sending $\lambda$ to 0 , finally sending $m$ to $+\infty$, we conclude that

$$
\limsup _{\sigma \rightarrow+\infty} \frac{\mu(\sigma A)}{\sigma^{2 / 3}} \leqslant \inf _{u \in \mathcal{W}\left(R^{2}\right)} \frac{\int_{\mathbb{R}^{2}}\left\{|\nabla u|^{2}+\frac{1}{4}\left|\operatorname{curl}^{2} A(0)\right|^{2}|x|^{4}|u|^{2}\right\} d x}{\int_{R^{2}}|u|^{2} d x} \leqslant p(1)\left|\operatorname{curl}^{2} A(0)\right|^{2 / 3} .
$$

So, (6.5) is true.

Now, we assume $0 \in \mathcal{Z}(A, \partial \Omega)$. From (3.11) it follows that

$$
\mathbf{a}(y)=A(0)+\nabla_{y} \tilde{\xi}(y)+\nabla_{y} \tilde{\zeta}(y)-\frac{1}{2}|y|^{2} \operatorname{curl}^{2} A(0)+\widetilde{D}(y) .
$$

Using the similar argument we obtain (6.6).

Remark 6.2: If there exist a smooth open subdomain $D \subset \Omega$ such that curl $A(x)$ vanishes in $D$, then

$$
\mu(\sigma A) \leqslant \inf _{\phi \in W_{0}^{1,2}(D)} \frac{\left\|\nabla_{\sigma A} \phi\right\|_{L^{2}(D)}^{2}}{\|\phi\|_{L^{2}(D)}^{2}}=\inf _{\phi \in W_{0}^{1,2}(D)} \frac{\|\nabla \phi\|_{L^{2}(D)}^{2}}{\|\phi\|_{L^{2}(D)}^{2}}=\lambda_{1}(D),
$$

where $\lambda_{1}(D)$ is the first Dirichlet eigenvalue of $-\Delta$ on $D$.

\section{LOWER-BOUND ESTIMATES}

In this section we give an lower bound of $\mu(\sigma A) /|\sigma|$ for large $\sigma$. The asymptotic behavior of the eigenfunctions as $\sigma \rightarrow \infty$ will also be discussed.

Lemma 7.1: Let $\Omega$ be a smooth bounded domain in $\mathbb{R}^{2}$ and $A \in C^{2}(\bar{\Omega})$. Then,

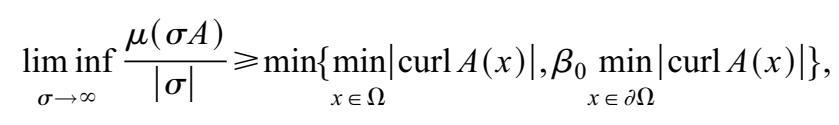

where $\beta_{0}$ is the positive constant given in Lemma 2.2 .

Proof: Let $H(x)=\operatorname{curl} A(x)$,

$$
\begin{gathered}
m(\Omega)=\min _{\bar{\Omega}}|H(x)|, \quad \Omega_{m}=\{x \in \Omega:|H(x)|=m(\Omega)\}, \\
m(\partial \Omega)=\min _{\partial \Omega}|H(x)|, \quad(\partial \Omega)_{m}=\{x \in \partial \Omega:|H(x)|=m(\partial \Omega)\}
\end{gathered}
$$


and $m=\min \left\{m(\Omega), m(\partial \Omega) \beta_{0}\right\}$. We shall show that $\lim _{\inf }{ }_{\sigma \rightarrow \infty} \mu(\sigma A) /|\sigma| \geqslant m$. As in Sec. VI we assume $\sigma>0$ and denote $\delta=1 / \sqrt{\sigma}$. Let $\psi^{\delta}$ be the eigenfunction associated with $\mu(\sigma A)$ satisfying $\max _{x \in \Omega}\left|\psi^{\delta}(x)\right|=1$. Then, $\psi^{\delta}$ satisfies

$$
\begin{gathered}
-\nabla_{\sigma A}^{2} \psi=\mu(\sigma A) \psi \quad \text { in } \Omega, \\
\left(\nabla_{\sigma A} \psi\right) \cdot \nu+\gamma \psi=0 \quad \text { on } \partial \Omega .
\end{gathered}
$$

Denote by $x^{\delta}$ the maximum point of $\left|\psi^{\delta}\right|$.

Now, we assume that $\left\{\sigma_{k}\right\}$ is a given sequence, $\sigma_{k} \rightarrow+\infty$. We choose a subsequence $\sigma_{k_{j}}$ such that

$$
x^{\delta_{k_{j}}} \rightarrow x^{0}, \quad \frac{\mu\left(\sigma_{k_{j}} A\right)}{\sigma_{k_{j}}} \rightarrow a
$$

for some non-negative number $a$. Lemma 6.1 implies $a \leqslant m$. We shall show $a \geqslant m$. Then it follows that $a=m$ for any sequence $\left\{\sigma_{k}\right\}$. For simplicity, we denote $\sigma_{k_{j}}$ by $\sigma$. Let $h_{\delta}=H\left(x^{\delta}\right), h$ $=H\left(x^{0}\right)$.

Case 1: $x^{0} \in \Omega$. We shall show $a \geqslant m(\Omega)$. Let $\Omega_{\delta}=\left(\Omega-x^{\delta}\right) / \delta, \quad \psi_{\delta}(x)=\psi^{\delta}\left(x^{\delta}+\delta x\right)$, $A_{\delta}(x)=(1 / \delta) A\left(x^{\delta}+\delta x\right), H_{\delta}(x)=H\left(x^{\delta}+\delta x\right)$. Note that $\operatorname{curl} A_{\delta}(x)=H_{\delta}(x)$. Using (7.2) we check that $\psi_{\delta}$ satisfies

$$
-\nabla_{A_{\delta}}^{2} \psi_{\delta}=\frac{\mu(\sigma A)}{\sigma} \psi_{\delta} \quad \text { in } \Omega_{\delta}
$$

and $\left|\psi_{\delta}(0)\right|=1=\left\|\psi_{\delta}\right\|_{L^{\infty}}$. We shall show that $\left\{\psi_{\delta}\right\}$ locally converges up to gauge transformations.

Let $R>0$ be a fixed constant. Then, for $\delta$ small enough we have $B_{3 R} \subset \Omega_{\delta}$. Since $\left\{\left|\psi_{\delta}\right|\right\}$ is uniformly bounded in $L_{\text {loc }}^{2}$, Lemma 4.2 implies that $\left\{\left|\nabla_{A_{\delta}} \psi_{\delta}\right|\right\}$ is also uniformly bounded in $L_{\text {loc }}^{2}$. Applying Theorem 4.1 to Eq. (7.3) we have

$$
\begin{aligned}
\left\|\left|\nabla_{A_{\delta}} \psi\right|\right\|_{H^{1}\left(B_{R}\right)}^{2} \leqslant & 2\left\|\psi_{\delta} H_{\delta}\right\|_{L^{2}(2 R)}^{2}+6\left[\frac{\mu(\sigma A)}{\sigma}\right]^{2}\left\|\psi_{\delta}\right\|_{L^{2}\left(B_{2 R}\right)}^{2} \\
& +C(R)\left[1+\left\|H_{\delta}\right\|_{L^{2}\left(B_{2 R}\right)}^{4}\right]\left[\left\|\nabla_{A_{\delta}} \psi_{\delta}\right\|_{L^{2}\left(B_{2 R}\right)}^{2}+\left\|\psi_{\delta}\right\|_{L^{2}\left(B_{2 R}\right)}^{2}\right] \\
\leqslant & C\left(R,\|H\|_{\left.L^{\infty}\right)}\left\|\psi_{\delta}\right\|_{L^{2}\left(B_{3 R}\right)}^{2} .\right.
\end{aligned}
$$

So, $\left\{\left|\nabla_{A_{\delta}} \psi_{\delta}\right|\right\}$ is uniformly bounded in $W_{\text {loc }}^{1,2}$, hence, is relatively compact in $L_{\text {loc }}^{2}$. Since $|\nabla| \psi_{\delta}||$ $\leqslant\left|\nabla_{A_{\delta}} \psi\right|,\left\{|\nabla| \psi_{\delta} \mid\right\}$ is uniformly bounded in $L_{\text {loc }}^{2}$. Thus, $\left\{\left|\psi_{\delta}\right|\right\}$ is relatively compact in $L_{\text {loc }}^{2}$ Passing to a subsequence we may assume that $\left|\psi_{\delta}\right|$ converges in $L_{\mathrm{loc}}^{2}\left(\mathbb{R}^{2}\right)$ as $\delta \rightarrow 0$. It follows from Lemma 3.1 that

$$
A_{\delta}(x)=\nabla_{\delta}(x)+h_{\delta} \omega(x)+B_{\delta}(x),
$$

where

$$
\chi_{\delta}(x)=\frac{1}{\delta} A\left(x^{\delta}\right) x+\frac{1}{2}\left[\partial_{1} A^{1}\left(x^{\delta}\right) x_{1}^{2}+\left(\partial_{1} A^{2}\left(x^{\delta}\right)+\partial_{2} A^{1}\left(x^{\delta}\right)\right) x_{1} x_{2}+\partial_{2} A^{2}\left(x^{\delta}\right) x_{2}^{2}\right]
$$

Set $\phi_{\delta}(x)=\exp \left(-i \chi_{\delta}\right) \psi_{\delta}(x)$. Then, $\phi_{\delta}$ satisfies

$$
-\nabla_{h \omega}^{2} \phi_{\delta}=\frac{\mu(\sigma A)}{\sigma} \phi_{\delta}+f_{\delta}(x)
$$


where $h=H\left(x^{0}\right)$ and

$$
\begin{aligned}
f_{\delta}(x)= & -\left[i \operatorname{div} B_{\delta}+2 h\left(h_{\delta}-h\right)+2 h \omega \cdot B_{\delta}+\left|\left(h_{\delta}-h\right) \omega+B_{\delta}\right|^{2}\right] \phi_{\delta} \\
& -2 i\left[\left(h_{\delta}-h\right) \omega+B_{\delta}(x)\right] \cdot \nabla \phi_{\delta} .
\end{aligned}
$$

Since $\left|\nabla_{h_{\delta} \omega+B_{\delta}} \phi_{\delta}\right|=\left|\nabla_{A_{\delta}} \psi_{\delta}\right|$ and

$$
\left|\nabla \phi_{\delta}\right|^{2} \leqslant\left|\nabla_{h_{\delta} \omega+B_{\delta}} \phi_{\delta}+i\left(h_{\delta} \omega+B_{\delta}\right) \phi_{\delta}\right|^{2} \leqslant 2\left|\nabla_{h_{\delta} \omega+B_{\delta}} \phi_{\delta}\right|^{2}+2\left|\left(h_{\delta} \omega+B_{\delta}\right) \phi_{\delta}\right|^{2},
$$

$\left\{\left|\nabla \phi_{\delta}\right|\right\}$ is also uniformly bounded in $L_{\text {loc }}^{2}$. Passing to another subsequence we have $\phi_{\delta} \rightarrow \phi_{0}$ weakly in $W_{\text {loc }}^{1,2}$ and strongly in $L_{\text {loc }}^{2}$. Since $\operatorname{div} B_{\delta}(x)=(\operatorname{div} A)\left(x^{\delta}+\delta x\right)-(\operatorname{div} A)\left(x^{\delta}\right) \rightarrow 0,\left|B_{\delta}(x)\right|$ $\leqslant C \delta|x|^{2}$ and $h_{\delta} \rightarrow h$, we have $f_{\delta} \rightarrow 0$ in $L_{\text {loc }}^{2}$. Hence, the limiting function $\phi_{0}$ satisfies

$$
-\nabla_{h \omega}^{2} \phi_{0}=a \phi_{0} \quad \text { in } R^{2}
$$

and $\left|\phi_{0}(x)\right| \leqslant 1$. Applying Theorem 4.1 to Eq. (7.5) yields that $\phi_{0}$ is smooth.

Denote $\hat{\phi}_{\delta}(x)=\phi_{\delta}(x)-\phi_{0}(x)$. From (7.4) and (7.5),

$$
-\nabla_{h \omega}^{2} \hat{\phi}_{\delta}=a \hat{\phi}_{\delta}+\hat{f}_{\delta}
$$

where

$$
\hat{f}_{\delta}=f_{\delta}+\left[\frac{\mu(\sigma A)}{\sigma}-a\right] \phi_{\delta} \rightarrow 0 \quad \text { in } L_{\mathrm{loc}}^{2}
$$

and $\hat{\phi}_{\delta} \rightarrow 0$ in $L_{\text {loc }}^{2}$. Applying Lemma 4.2 to (7.6) we get $\left|\nabla_{h \omega} \hat{\phi}_{\delta}\right| \rightarrow 0$ in $L_{\text {loc }}^{2}$. Since $\left|\nabla \hat{\phi}_{\delta}\right|^{2}$ $\leqslant 2\left|\nabla_{h \omega} \hat{\phi}_{\delta}\right|^{2}+2\left|h \omega \hat{\phi}_{\delta}\right|^{2}$ we have $\left|\nabla \hat{\phi}_{\delta}\right| \rightarrow 0$ in $L_{\text {loc }}^{2}$. So,

$$
\hat{\phi}_{\delta} \rightarrow 0 \quad \text { in } W_{\text {loc }}^{1,2} \text {. }
$$

Denote $\omega=\left(\omega^{1}, \omega^{2}\right), \nabla_{h \omega^{j}}=\partial_{j}-i h \omega^{j}$. Applying Theorem 4.1 to Eq. (7.6) we have

$$
\nabla_{h \omega^{j}} \nabla_{h \omega^{k}} \hat{\phi}_{\delta} \rightarrow 0 \quad \text { in } L_{\text {loc }}^{2}
$$

Note that, for example,

$$
\nabla_{h \omega^{1}} \nabla_{h \omega^{1}} \hat{\phi}_{\delta}=\frac{\partial^{2}}{\partial x_{1}} \hat{\phi}_{\delta}+i h x_{2} \frac{\partial}{\partial x_{1}} \hat{\phi}_{\delta}-\frac{1}{4}\left|h x_{2}\right|^{2} \hat{\phi}_{\delta} .
$$

Therefore, (7.7) and (7.8) imply that $\partial_{j} \partial_{k} \hat{\phi}_{\delta} \rightarrow 0$ in $L_{\mathrm{loc}}^{2}$. So, $\hat{\phi}_{\delta} \rightarrow 0$ in $L_{\mathrm{loc}}^{2}$.

Now, we apply the classical $C^{\alpha}$ estimates to (7.6) and conclude that $\hat{\phi}_{\delta} \rightarrow 0$ in $C_{\text {loc }}^{\alpha}$, that is, $\phi_{\delta} \rightarrow \phi_{0}$ in $C_{\mathrm{loc}}^{\alpha}$. Especially, we get $\phi_{0}(0)=\lim _{\delta \rightarrow 0} \phi_{\delta}(0)=1$. Therefore, $\phi_{0}$ is a nonzero bounded smooth solution of Eq. (7.5) in $\mathbb{R}^{2}$. From Lemma 2.1 we have

$$
a \geqslant \alpha(h)=|h|=\left|H\left(x^{0}\right)\right| \geqslant \min _{x \in \Omega}|H(x)|=m(\Omega) .
$$

Since $a \leqslant m$, we conclude that $a=m$. We also see that if Case 1 happens then $m=m(\Omega), x^{0}$ $\in \Omega_{m}$ and (7.1) holds.

Case 2: $x^{0} \in \partial \Omega$. Now, we shall prove $a \geqslant m(\partial \Omega)$. Let $d_{\delta}=\operatorname{dist}\left(\partial \Omega, x^{\delta}\right)$, the distance between $x^{\delta}$ and $\partial \Omega$. Then, $B_{d_{\delta} / \delta} \subset \Omega_{\delta}$. If there exists a subsequence $\delta_{j} \rightarrow 0$ such that $d_{\delta_{j}} / \delta_{j} \rightarrow \infty$, then the argument in Case 1 also gives that $\mu\left(\sigma_{j} A\right) /\left|\sigma_{j}\right| \rightarrow\left|H\left(x_{0}\right)\right|$. Therefore, we assume that $d_{\delta} / \delta$ is bounded. Passing to a subsequence, we may assume that $d_{\delta} / \delta \rightarrow d_{0}$. 
Let $\hat{x}^{\delta} \in \partial \Omega$ such that $\left|\hat{x}^{\delta}-x^{\delta}\right|=\operatorname{dist}\left(x^{\delta}, \partial \Omega\right)=d_{\delta}$. At each point $\hat{x}^{\delta}$ we take a diffeomorphism $\mathcal{F}_{\delta}: B_{R_{0}}^{+} \rightarrow \Omega \cap \mathcal{F}_{\delta}\left(B_{R_{0}}\right)$ to straighten a portion of boundary around the point $\hat{x}^{\delta}$ such that $\mathcal{F}_{\delta}(0)=\hat{x}^{\delta}$. For simplicity, we denote $\mathcal{F}_{\delta}$ by $\mathcal{F}$. We keep in mind that the diffeomorphism $\mathcal{F}$ depends on $\delta$. However, the constant $R_{0}$ can be chosen to be independent of $\delta$, thus, we have uniform estimates on $\mathcal{F}$ for all small $\delta$. Let $y^{\delta} \in B_{R_{0}}^{+}$be such that $\mathcal{F}\left(y^{\delta}\right)=x^{\delta}$. Then, $\left|y^{\delta}\right| \leqslant C d_{\delta}$ $\leqslant C \delta$.

Let $\psi^{\delta}(\mathcal{F}(y))=u(\mathcal{F}(y)) \tilde{\psi}^{\delta}(y)$, where $u$ is the positive eigenfunction of Eq. (5.2) associated with the first eigenvalue $\lambda$ and $\|u\|_{L_{\infty}}=1$. Then, $\left\{\left|\tilde{\psi}^{\delta}\right|\right\}$ is uniformly bounded from above and $\left|\tilde{\psi}^{\delta}\left(y^{\delta}\right)\right|$ is uniformly bounded away from zero. As in Sec. V we can check that $\tilde{\psi}^{\delta}$ satisfies the following equation:

$$
\begin{gathered}
-\Delta(g)_{\sigma \mathbf{a}} \widetilde{\psi}^{\delta}=D(g) \widetilde{v} \cdot D(g)_{\sigma \mathbf{a}} \widetilde{\phi}^{\delta}+[\mu(\sigma A)-\lambda] \widetilde{\psi}^{\delta} \text { in } B_{R_{0}}^{+}, \\
\left(D(g)_{\sigma \mathbf{a}} \widetilde{\psi}^{\delta}\right) \cdot \nu=0 \quad \text { on } \Gamma_{R_{0}} .
\end{gathered}
$$

Here the notations involved are the same as in Secs. III and V.

Define the following rescaled functions and vector fields: $\widetilde{\psi}_{\delta}(y)=\widetilde{\psi}^{\delta}(\delta y), \widetilde{v}_{\delta}(y)=\widetilde{v}(\delta y)$, $g_{\delta}(y)=g(\delta y), \mathbf{a}_{\delta}(y)=(1 / \delta) \mathbf{a}(\delta y)$. Then,

$$
\begin{gathered}
-\Delta\left(g_{\delta}\right)_{\mathbf{a}_{\delta}} \tilde{\psi}_{\delta}=D\left(g_{\delta}\right) \tilde{v}_{\delta} \cdot D\left(g_{\delta}\right)_{\mathbf{a}_{\delta}} \tilde{\psi}_{\delta}+\frac{\mu(\sigma A)-\lambda}{\sigma} \tilde{\psi}_{\delta} \text { in } B_{R_{0} / \delta}^{+}, \\
\left(D\left(g_{\delta}\right)_{\mathbf{a}_{\delta}} \tilde{\psi}_{\delta}\right) \cdot \nu=0 \quad \text { on } \Gamma_{R_{0} / \delta} .
\end{gathered}
$$

Recall that $h_{\delta}=H\left(x^{\delta}\right), h=H\left(x^{0}\right)$. From (3.11) we have

$$
\mathbf{a}_{\delta}(y)=\nabla_{y} \tilde{\chi}_{\delta}(y)+h_{\delta} \widetilde{\omega}(y)+\widetilde{B}_{\delta}(y),
$$

which holds in $B_{R_{0} / \delta}^{+}$, but not in the entire ball. Set $\widetilde{\phi}_{\delta}(y)=\exp \left(-i \tilde{\chi}_{\delta}\right) \widetilde{\psi}_{\delta}$. Then,

$$
-\Delta\left(g_{\delta}\right)_{h_{\delta} \tilde{\omega}+\tilde{B}_{\delta}} \widetilde{\phi}_{\delta}=D\left(g_{\delta}\right) \widetilde{v}_{\delta} \cdot D\left(g_{\delta}\right)_{h_{\delta} \tilde{\omega}+\tilde{B}_{\delta}} \widetilde{\phi}_{\delta}+\frac{\mu(\sigma A)-\lambda}{\sigma} \widetilde{\phi}_{\delta} \text { in } B_{R_{0} / \delta}^{+} .
$$

Using Theorem 5.5 we obtain

$$
\widetilde{\phi}_{\delta} \rightarrow \widetilde{\phi}_{0} \text { weakly in } W_{\text {loc }}^{1,2} \text { and strongly in } L_{\text {loc }}^{2} \text {. }
$$

Write $\widetilde{B}_{\delta}=\widetilde{B}_{\delta}^{1} \mathbf{e}_{1}+\widetilde{B}_{\delta}^{2} \mathbf{e}_{2}$, and write the equation for $\widetilde{\phi}$ as follows:

$$
\begin{gathered}
-\Delta\left(g_{\delta}\right)_{h \tilde{\omega}} \widetilde{\phi}_{\delta}=\frac{\mu(\sigma A)}{\sigma} \widetilde{\phi}_{\delta}+\widetilde{f}_{\delta} \quad \text { in } B_{R_{0} / \delta}^{+}, \\
\left(D\left(g_{\delta}\right)_{h_{\delta \tilde{\omega}}} \widetilde{\phi}_{\delta}\right) \cdot \nu=-i \widetilde{B}_{\delta}^{2} \widetilde{\phi}_{\delta} \quad \text { on } \Gamma_{R_{0} / \delta} .
\end{gathered}
$$

Note that $D\left(g_{\delta}\right) \widetilde{v}_{\delta} \cdot D\left(g_{\delta}\right)_{h_{\delta} \tilde{\omega}+\tilde{B}_{\delta}} \widetilde{\phi}_{\delta} \rightarrow 0$ in $L_{\text {loc }}^{2}$ and $\widetilde{B}_{\delta} \rightarrow 0$ in $L_{\text {loc. }}^{2}$. So, $\widetilde{f}_{\delta} \rightarrow 0$ in $L_{\text {loc. }}^{2}$. We also note that $g_{\delta}(y)=1-\delta y_{2} \kappa_{r}\left(\delta y_{1}\right) \rightarrow 1$. Hence, $\widetilde{\phi}_{0}$ satisfies

$$
\begin{gathered}
-\nabla_{h \omega}^{2} \widetilde{\phi}_{0}=\alpha \widetilde{\phi}_{0} \quad \text { in } \mathbb{R}_{+}^{2}, \\
\nabla_{h \omega} \cdot \widetilde{\phi}_{0}=0 \text { on } \partial R_{+}^{2} .
\end{gathered}
$$


We apply Theorem 5.5 to the equation for $\tilde{\phi}-\widetilde{\phi}_{0}$, then use the classical elliptic estimates to obtain that $\widetilde{\phi}_{\delta} \rightarrow \widetilde{\phi}_{0}$ in $C_{\text {loc }}^{\alpha}$.

Recall that $\left|y^{\delta}\right| / \delta \leqslant C$. By passing to a subsequence, we may assume that $z^{\delta} \equiv y^{\delta} / \delta \rightarrow z^{0}$, hence, $\widetilde{\phi}_{0}\left(z_{0}\right)=\lim \widetilde{\phi}_{\delta}\left(z^{\delta}\right) \neq 0$. Therefore, $\widetilde{\phi}_{0} \neq 0$, that is, $\widetilde{\phi}_{0}$ is a nonzero bounded smooth solution of Eq. (7.11). Using Lemma 2.2 we conclude that

$$
a \geqslant \beta(h)=\beta_{0}|h|=\beta_{0}\left|H\left(x^{0}\right)\right| \geqslant \beta_{0} \min |H(x)|=m(\partial \Omega) \beta_{0} .
$$

Since $m(\partial \Omega) \beta_{0} \geqslant m \geqslant a$, we have $m=a$. We also see that if Case 2 happens then $m$ $=m(\partial \Omega) \beta_{0}, x^{0} \in(\partial \Omega)_{m}$ and (7.1) holds.

The proof of Lemma 7.1 has the following consequence.

Proposition 7.2: Assume that $A \in C^{2}(\bar{\Omega})$. Let $\psi_{\sigma}$ be the eigenfunction of Eq. (7.2) associated with the first eigenvalue $\mu(\sigma A)$ such that $\left\|\psi_{\sigma}\right\|_{L_{\infty}} \leqslant C$. Then,

$$
\left|\psi_{\sigma}\right| \rightarrow 0 \quad \text { in } C_{\mathrm{loc}}^{\alpha}\left(\Omega \backslash \Omega_{m}\right) \text { and in } C_{\mathrm{loc}}^{\alpha}\left(\partial \Omega \backslash(\partial \Omega)_{m}\right) \text { as } \sigma \rightarrow \infty .
$$

Proof of Theorem 1: Combining Lemmas 6.1, 6.2, 7.1 yields Theorem 1.

As a corollary of Theorem 1 and Proposition 7.2, we have

Theorem 7.3: Assume $A \in C^{2}(\bar{\Omega})$ and $\operatorname{curl} A(x) \equiv H$, a nonzero constant. Then

$$
\lim _{\sigma \rightarrow \infty} \frac{\mu(\sigma A)}{|\sigma|}=\beta_{0}|H|
$$

Let $\psi_{\sigma}$ be the eigenfunction of (7.2) satisfying $\left\|\psi_{\sigma}\right\|_{L_{\infty}}=1$, then $\psi_{\sigma}$ concentrates at some points on $\partial \Omega$, that is, $\left\|\psi_{\sigma}\right\|_{L_{\infty}(\partial \Omega)} \rightarrow 1$ and

$$
\psi_{\sigma} \rightarrow \text { in } C^{\alpha}(\Omega) \text { as } \sigma \rightarrow \infty .
$$

Proposition 7.2 says that as $\sigma \rightarrow \infty$ the eigenfunctions concentrate at some points in $\Omega_{m} \cup(\partial \Omega)_{m}$. From the proof of Lemma 7.1 one easily see that, after rescaling near the maximum points and making gauge transitions, the eigenfunctions exhibit profiles of either the eigenfunction of (2.3) in the entire plane $\mathbb{R}^{2}$ (when interior concentration happens), or the eigenfunction of (2.5) in the half plane $\mathbb{R}_{+}^{2}$ (when boundary concentration happens). It will be interesting to find the exact location of the concentration points. In Ref. 1 the concentration behavior of minimal solutions of Ginzburg-Landau equations is studied and the location of concentration is investigated. The arguments used in Ref. 1 can be applied in a similar way to obtain the location of concentration of the eigenfunctions. We should mention that in Ref. 10 Bernoff and Sternberg obtained the location of surface nucleation of superconductivity by using the asymptotic analysis.

\section{ACKNOWLEDGMENTS}

This work was partially done when the second author (X.-B.P.) visited the Department of Mathematics at Brigham Young University in the academic year of 1995. He would like to thank the Department for hospitality. This work was partially supported by National Science Foundation Grant No. DMS-9622853 (to K.L.) and by Chinese National Natural Science Foundation, Science Foundation of the Ministry of Education of China, and Zhejiang Provincial Natural Science Foundation of China (to X.-B.P.)

\footnotetext{
${ }^{1}$ K. Lu and X.-B. Pan, “Estimates of upper critical external magnetic field for the Ginzburg-Landau equation,” Physica D 127, 73-104 (1999).

${ }^{2}$ D. Saint-James and P. de Gennes, “Onset of superconductivity in decreasing fields," Phys. Lett. 7, 306-308 (1963).

${ }^{3}$ S. J. Chapman, "Nucleation of superconductivity in decreasing fields, 1, 2," Eur. J. Appl. Math. 5, 449-468 (1994); 5, 469-494 (1994).

${ }^{4} \mathrm{~K}$. Lu and X.-B. Pan, "Gauge invariant eigenvalue problems in $\mathbb{R}^{2}$ and in $\mathbb{R}_{+}^{2}$, , Trans. Am. Math. Soc. (to be published).
} 
${ }^{5}$ V. Ginzburg and L. Landau, “On the theory of superconductivity,” Sov. Phys. JETP 20, 1064-1082 (1950).

${ }^{6} \mathrm{P}$. De Gennes, Superconductivity of Metals and Alloys (Benjamin, New York, 1966).

${ }^{7}$ P. Bauman, D. Phillips, and Q. Tang, "Stable nucleation for the Ginzburg-Landau system with an applied magnetic field," Arch. Ration. Mech. Anal. 142, 1-43 (1998).

${ }^{8}$ F. Bethuel, H. Brézis, and F. Hélein, Ginzburg-Landau Vortices (Birkhöuser, Boston, 1994).

${ }^{9} \mathrm{~F}$. Bethuel and T. Riviére, "Vortices for a variational problem related to superconductivity," Ann. Inst. Henri Poincaré Anal. Non Linéaire 12, 243-303 (1995).

${ }^{10}$ A. Bernoff and P. Sternberg, "'Onset of superconductivity in decreasing fields for general domains," J. Math. Phys. 39, 1272-1284 (1998).

${ }^{11}$ S. J. Chapman, S. D. Howison, and J. R. Ockendon, “Macroscopic models for superconductivity,” SIAM (Soc. Ind. Appl. Math.) Rev. 34, 529-560 (1992).

${ }^{12}$ S. Chanillo and M. Kiessling, "Symmetry of solutions of Ginzburg-Landau equations," Comp. Rend. Acad. Sci., Paris, 32, 1023-1026 (1995).

${ }^{13} \mathrm{Q}$. Du, M. Gunzburger, and J. Peterson, "Analysis and approximation of the Ginzburg-Landau model of superconductivity,” SIAM (Soc. Ind. Appl. Math.) Rev. 34, 45-81 (1992).

${ }^{14}$ A. Jaffe and C. Taubes, Vortices and Monopoles (Birkhäuser, Boston, 1980).

${ }^{15}$ F.-H. Lin, "Solutions of Ginzburg-Landau equations and critical points of the renormalized energy," Ann. Inst. Henri Poincaré, Anal. Non Linéaire 12, 599-622 (1995).

${ }^{16}$ F.-H. Lin and T.-C. Lin, "Minimax solutions of the Ginzburg-Landau equations," Selecta Math. (N.S.) 3, 99-113 (1997).

${ }^{17}$ K. Lu and X.-B. Pan, “Ginzburg-Landau equation with DeGennes boundary condition,” J. Diff. Eqns. 129, 136-165 (1996).

${ }^{18}$ P. Mironescu, "On the stability of radial solutions of the Ginzburg-Landau equations," J. Funct. Anal. 130, 334-347 (1995).

${ }^{19}$ M. Struwer, "On the asymptotical behavior of minimizers of the Ginzburg-Landau model in 2-dimensions," J. Diff. Eqns. 7, 1613-1627 (1994). 